Cinémas

Revue d'études cinématographiques

Journal of Film Studies

\title{
Comment éditer Eisenstein? Problème de Méthode (extraits inédits)
}

\section{Oksana Boulgakova}

Volume 11, numéro 2-3, printemps 2001

Eisenstein dans le texte

URI : https://id.erudit.org/iderudit/024846ar

DOI : https://doi.org/10.7202/024846ar

Aller au sommaire du numéro

Éditeur(s)

Cinémas

ISSN

1181-6945 (imprimé)

1705-6500 (numérique)

Découvrir la revue

Citer cet article

Boulgakova, O. (2001). Comment éditer Eisenstein? Problème de Méthode (extraits inédits). Cinémas, 11(2-3), 27-67. https://doi.org/10.7202/024846ar

\section{Résumé de l'article}

Cette étude s'efforce de présenter le dernier des trois grands projets théoriques, restés inachevés et non publiés à la mort d'Eisenstein, Méthode. Le maître soviétique cherchait à établir un " système théorique absolu - et utopique " qu'il entrevoyait de différentes manières. D'abord en tant que combinatoire artistique relevant d'un montage, qui donne lieu au projet d'un " livre sphérique », en 1929. Puis en tant que correspondance globale entre les sens et leur matérialisation dans l'art (son et couleur, synesthésie du contrepoint audio/visuel), explorée dans Montage (1937-1940). Enfin, en tant que " méthode artistique universelle appliquée à l'art ", méthode universelle d'analyse permettant d'approcher tous les phénomènes examinés dans Méthode (1939-1947), où il s'attaque à un matériau plus vaste. La présente étude comprend une vingtaine de pages du dernier projet et examine les problèmes complexes que pose sa publication. 


\section{Comment éditer Eisenstein? \\ Problème de Méthode \\ (extraits inédits)}

\section{Oksana Boulgakova}

\section{RÉSUMÉ}

Cette étude s'efforce de présenter le dernier des trois grands projets théoriques, restés inachevés et non publiés à la mort d'Eisenstein, Méthode. Le maître soviétique cherchait à établir un "système théorique absolu - et utopique " qu'il entrevoyait de différentes manières. D'abord en tant que combinatoire artistique relevant d'un montage, qui donne lieu au projet d'un "livre sphérique", en 1929. Puis en tant que correspondance globale entre les sens et leur matérialisation dans l'art (son et couleur, synesthésie du contrepoint audio/visuel), explorée dans Montage (1937-1940). Enfin, en tant que "méthode artistique universelle appliquée à l'art ", méthode universelle d'analyse permettant d'approcher tous les phénomènes examinés dans $M e ́-$ thode (1939-1947), où il s'attaque à un matériau plus vaste. La présente étude comprend une vingtaine de pages du dernier projet et examine les problèmes complexes que pose sa publication.

\section{ABSTRACT}

This article introduces Method, the last of Eisenstein's three rnajor theoretical projects, which remained incomplete and unpublished at his death. Here Eisenstein attempted to establish an "absolute" and "utopian" theoretical system, which he understood in different ways. First of all, as an arristic device, proceeding from montage, which inspired his ambition in 1929 to produce a "spherical book". Second, as an overall correspondence 
between the senses and their materialisarion in art (e.g., sound and colour; the synaesthetic quality of audiovisual counterpoint), which he explored in Montage (1937-40). Finally, as a "universal artistic method", or a universal method of analysis, which would enable him to unite the vast range of material taken up in Method (1939-47). The present article includes some twenty pages from this final project and examines the complex problems posed by its publication.

Parmi les projets d'une théorie globale non seulement du cinéma mais de l'art, de la personnalité et de la conscience, le dernier livre d'Eisenstein, Méthode, semble être le plus énigmatique. En dépit de l'intérêt que lui ont manifesté plusieurs chercheurs qui lui ont consacré articles et livres ${ }^{1}$, ce manuscrit de quelque 2459 feuillets recto-verso n'a pas encore été publié et n'est connu que par quelques extraits épars ${ }^{2}$. Encore l'attribution de certains textes comme appartenant à Méthode peut-elle être contestée ${ }^{3}$.

C'est, de toute évidence, un an après son retour du Mexique qu'Eisenstein a commencé à travailler sur ce livre; de cette époque date le premier plan de travail. Le 24 mars 1933, il fait une première ébauche de préface et compose un plan qu'il suit jusqu'aux dernières ébauches de 1948. Le livre change de titre, mais son plan et sa conception ne subissent pas de changements essentiels. Dans le contexte des autres projets d'Eisenstein (le "livre sphérique" de 1929 et Montage de 1937-1940), l'idée principale de Méthode, malgré un matériel pléthorique, est, à l'origine, réduite. Pour comprendre son originalité, je me permettrai de présenter brièvement deux autres conceptions de la théorie "totale" de l'art.

\section{La théorie comme projet utopique}

À la fin des années vingt, Eisenstein forme un premier projet de livre, le "livre sphérique». D'une part, il souhaite présenter sa position sans passer par un médiateur comme Alexandre Belenson en $1924^{4}$, d'autre part, entre 1926 et 1929 , sortent les livres de ses "rivaux" — Timochenko, Poudovkine, les formalistes, Kouléchov ${ }^{5}$. Eisenstein s'efforce de briser par son livre les formes traditionnelles d'exposition de la théorie sous l'espèce du manifeste, de l'article ou du livre, de la classification des techni- 
ques de montage, de l'archaïque Mon expérience dans l'art ou de l'essai théorique académique.

Il est très difficile d'écrire un livre, note-t-il dans son "Journal" le 5 août 1929, parce que chaque livre a deux dimensions. J'aimerais que celui-ci se distingue par une qualité qui n'entre pas dans la nature bidimensionnelle de l'ouvre imprimée. Cette exigence est double. D'une part, le recueil de ces essais ne doit absolument pas être reçu linéairement : je souhaite une réception simultanée de tous en même temps, parce qu'à la fin, ils sont tous différents secteurs de différents domaines rassemblés autour d'un seul point central qui les définit tous - par la méthode. D'autre part j'aimerais établir, d'un point de vue purement spatial, la possibilité que chaque texte entre en contact immédiat avec le texte voisin. [...] Seul un livre en forme de... sphère pourrait correspondre à une telle synchronie et une telle interpénétration des textes. Mais, hélas! les livres ne s'écrivent pas en sphères. [...] Il ne nous reste qu'à espérer que ce livre, écrit selon la méthode de la réversibilité muruelle infinie, sera lu selon la méthode sphérique. En attendant que nous apprenions à écrire et à lire les livres comme des sphères tournantes. Des livres qui ressemblent à des bulles de savon, il y en a assez aujourd'hui. Surtout sur l'art".

De mars à août 1929, Eisenstein compose le plan de ce livre? Il procède non seulement à une nouvelle organisation des articles sur le montage partiellement publiés ("Montage des attractions", "Perspectives", "Hors cadre", "La quatrième dimension au cinéma»), partiellement écrits et non publiés (comme "Montage des attractions au cinéma»), partiellement projetés et non écrits (comme "Khlebnikov", "Le pair", etc.) qui seraient réunis, mais à une nouvelle approche de l'organisation livresque et de la pensée en tant que montage non motivé de points de vue, de méthodes d'analyse et d'approches entre des secteurs communiquant librement entre eux.

On trouve des traces de ce projet dans la correspondance d'Eisenstein jusqu'en 1932. Le 23 août 1929, quatre jours après son arrivée à Berlin, il écrit à Kenneth MacPherson, l'éditeur de Close up qui a publié sa "Déclaration sur le son" et l'article "New Language of Cinematography ${ }^{8}$ ": "J'ai le manuscrit d'un 
livre que j'ai écrit sur la théorie du cinéma. Nous pourrions aussi en discuter..." (cité par Seton, 1952, p. 127).

Il écrit depuis le Mexique au même correspondant', en lui envoyant la traduction anglaise de "Dramaturgie der FilmForm » (ibid., p. 218):

J'espère que vous aimerez "Film-Form» - Je pense que c'est un des plus sérieux et des plus fondamentaux de ce que je pense sur le cinéma - et il peut être présenté comme une page de mon encore (et j'en ai peur à jamais!) livre "à venir»!

Des articles épars de ce livre ont été publiés. Qu'est-ce qui en a été perdu? Le montage, désigné comme "méthode" dans l'extrait d'Eisenstein, était analysé dans un «faisceau " d'articles dans différentes perspectives : la réflexologie (montage comme création de la chaîne de réflexes conditionnés par la combinaison aléatoire de stimuli: "Montage des attractions", "Montage des attractions au cinéma "); le constructivisme et le formalisme à ses débuts (montage comme combinaison, recombinaison et collage de matériaux différents : voir "Montage des attractions"); la hiéroglyphique ("Hors cadre"); la linguistique et la théorie du langage où celui-ci est traité selon le paradigme saussurien comme système d'oppositions qui forment l'énoncé ( «Perspectives»); la dialectique dans le cadre de la loi de l'unité des contraires ("Dramaturgie de la forme filmique»); mais aussi la musique et le théâtre japonais dans le cadre de la méditation sur la synchronisation des sens différents (la vision, l'audition, jusqu'à l'odorat, le toucher et le goût) ("La quatrième dimension au cinéma ", "Un point de jonction imprévu»), etc. Eisenstein change chaque fois de grille d'analyse en cherchant à obtenir ce changement de points de vue qui lui semblait particulièrement important à la fin des années vingt. C'est pourquoi le "constructiviste" et le "symboliste" synesthésique et métaphysique, le "matérialiste vulgaire" et le "réflexologue" sont présents en même temps que le "structuraliste» et le "dialecticien", manifestement sous influence de la Gestaltpsychologie. À ce propos, parallèlement à l'idée du "livre sphérique", Eisenstein projette d'écrire un livre d'auto-analyse sur lui-même et sur l'origine de sa théorie, My Art in Life ${ }^{10}$ - encore un rayon. 
Presque toutes les notions introduites par Eisenstein dans ce livre (attraction, dominante, harmonique, intervalle, image, montage) ont passé par des systèmes d'analyse et d'interprétation différents (ainsi, "dominante" renvoie en même temps à la théorie du texte de Tynianov, aux expériences musicales de Scriabine et Alban Berg et au traitement de ce terme dans la psychologie de William James), ce qui fait résonner différemment les textes du livre.

Le principe sphérique reflète la nouvelle situation dans la science du début du siècle, quand le monde harmonieux des systèmes clos s'est brisé. Les nouvelles découvertes en sciences naturelles ont amené à la pluralisation des discours scientifiques. La totalité a été rejetée comme utopie, mais les résultats des découvertes particulières sont interprétés, par habitude, comme une représentation générale du monde. Les théories ont une signification opératoire et fonctionnelle, l'ontologie est rejetée, l'analyse professionnelle est affirmée comme une restriction nécessaire et la multitude des différents types de discours n'embrasse que certains aspects du phénomène. Le "livre sphérique» est un produit de cette nouvelle pensée. D'un côté, Eisenstein se comporte comme un expert hautement spécialisé (comme les formalistes qui peuvent distinguer la poésie de la prose et même faire apparaître le "filmique" comme objet d'analyse), d'un autre côté, il s'efforce de créer un cadre unique pour des discours différents, en proposant cette forme sphérique qui suppose le libre passage de l'un à l'autre, la multitude de points de vue superposés sur le même plan (comme dans le cubisme). Ses premiers modèles de livre et de théorie sont des propositions radicales de créer un système qui n'en soit pas un, une totalité qui n'en soit pas une et suppose une réinterprétation continuelle de secteurs incompatibles.

Le deuxième projet, Montage, se formalise à la fin des années trente et le livre s'écrit entre 1937 et 1940 . Le projet de cette recherche surgit sous l'influence de l'Esthétique de Hegel, de l'Ulysse de Joyce, et de l'étude qu'en propose Stuart Gilbert sous la conduite de Joyce lui-même ${ }^{11}$, enfin de La Philosophie des formes symboliques d'Ernst Cassirer.

Ulysse a non seulement été envisagé comme un roman mais comme un livre universel de correspondances à des niveaux 
différents. Le lieu de l'action de tous les chapitres peut être retrouvé sur la carte de Dublin, mais en même temps, tous ensemble, ils représentent la carte d'une ville universelle et de la vie humaine en transformant le concret en métaphorique, le particulier en général - rencontre, séparation, etc. Chaque chapitre a son leitmotiv de couleurs, tous ensemble ils donnent le spectre complet et correspondent aux couleurs de la messe catholique. La plupart des chapitres symbolisent un organe particulier, le roman dans son ensemble étant une allégorie du corps. Chaque chapitre a son leitmotiv sémantique qui correspond à un type d'art particulier (renvoyant lui-même, à l'intérieur de la vision médiévale, à une science particulière). Ainsi, tout le roman est-il construit comme un symbole gigantesque et un compendium du savoir universel. Ce schéma de correspondances, qu'il avait communiqué à Stuart Gilbert, Joyce l'a montré à Eisenstein au moment de leur rencontre à Paris, en novembre 1930.

Lidée de publier un tel livre sur la théorie de l'art en cherchant des correspondances entre la parole et l'image, la ligne et la mélodie, le son et la couleur, la couleur et la forme, le visible et l'invisible et, plus largement, entre les différents arts - peinture, littérature, musique, architecture, cinéma et cirque - a longtemps occupé Eisenstein. Montage est devenu son Laocoon où, après Lessing, il évalue à nouveaux frais le rapport entre les arts plastiques et littéraires, parce que les arts visuels se lisent à travers l'expérience du cinéma, et le cinéma à travers l'expérience de l'esthétique philosophique qui étudie le rapport entre le subjectif et l'objectif. Eisenstein construit son livre non seulement sur les correspondances entre les différents arts, mais entre l'art, le corps et le cosmos qui sont vécus par le sujet percevant dans le processus de la perception de l'œuvre d'art qui transcende la dualité de la conscience et de l'être. Au cours de son analyse, Eisenstein cherche à formaliser des structures de composition inconscientes et - par analogie avec la notion de "symbole», telle que l'a réélaborée Cassirer qui, par là, a révolutionné l'ontologie - propose une conception de l'«image» qui se place au centre d'un nouveau système et devient la base de correspondances universelles. 
Au moment de l'écriture de ce livre, Eisenstein travaille à son premier film sonore, Alexandre Nevski, fait la mise en scène de la Walkyrie et a le projet de son premier film "en couleurs", L'Amour d'un poète; il travaille également à l'idée d'œuvre d'art totale qui synchronise tous les sens. Mais il part non de l'audition, comme Wagner, mais de la vision, laquelle peut être restructurée par les stimuli acoustiques, tactiles et autres.

Or Montage, ce livre gigantesque, plus volumineux que $M e^{-}$ thode, et où il inclut des extraits d'articles des années vingt en les réinterprétant à l'intérieur de sa nouvelle conception, reste, lui aussi, inachevé. Eisenstein l'abandonne pour un autre projet qui propose une autre conception utopique de la totalité. Ce troisième livre, Méthode, dont le projet apparaît avant Montage, s'élabore pourtant plus tard. Sa conception y semble très réductrice par rapport aux correspondances multipliées à l'infini de Montage. Méthode vise à l'universalisation à l'aide d'un modèle analytique simple applicable à n'importe quelle ouvre d'art, de n'importe quel type, de n'importe quelle époque: Disney et Bach, le cubisme et la peinture naturaliste, Outamaro et Degas, Dostö̈evski et le système de jeu selon Stanislavski, les romans policiers et les caricatures, les métaphores de Gœthe et les limericks anglais, ses propres mises en scène au thêâtre et au cinéma, ainsi qu'une cathédrale gothique ou la poésie de Baudelaire! Ce nouveau fondement universel est la structure de la conscience elle-même.

Au moment de la perception extatique, l'œuvre d'art provoque chez le sujet un passage à la pensée sensible, prélogique qui "brise" la logique comme l'inconscient freudien fracture la conscience. Eisenstein conçoit cependant cette situation comme un équilibre dynamique entre des réactions polarisées et poussées, d'un côté, vers les profondeurs du «sensible» et, de l'autre, vers le haut, vers les structures logiques. L'œuvre d'art est comprise comme une empreinte de la conscience, faite, pour ainsi dire, de plusieurs couches, mais il est également possible d'observer et d'analyser un tel phénomène sur d'autres empreintes matérielles (les réactions motrices des affects, les symboles visuels, les topos stables, etc.). Toutes ces «traces» sont envisagées comme des empreintes du conflit entre différents types de pensée. Pour les désigner, Eisenstein utilise les termes de "sensible » 
et de "rationnel». Il a d'abord recouru aux termes de "prélogique» et "logique », mais après que les théories de Lucien LévyBruhl, à qui il avait emprunté ces termes, eurent été vivement critiquées en Union soviétique, il les a remplacés par des termes plus neutres comme la "pensée sensible". Cependant Otto Rank avait déjà remarqué, en 1932, qu'il y avait beaucoup de correspondances entre l'" inconscient" de Freud, le "prélogique" chez Lévy-Bruhl et le "mythique " chez Cassirer ${ }^{12}$.

Là où Montage recherchait des correspondances entre son et ligne, couleur et son, Méthode cherche à formaliser les structures de l'inconscient via l'analyse de ses traces dans les formes et ses techniques, et arrive à l'idée d'une sémiotisation totale du monde. Par analogie avec la double lecture des symptômes primaires et secondaires dans l'herméneutique psychanalytique, Eisenstein transforme la loi de la dialectique de l'unité des contraires en système d'oppositions binaires (conscient/inconscient, logique/prélogique ou rationnel/sensible). C'est-à-dire que la multiplicité des formes se présente désormais comme une chaîne infinie d'invariants réductibles au conflit de base vécu par la pensée en devenir: le passage du prélogique au logique. À l'aide de ce modèle analytique simple, tous les phénomènes peuvent être décrits, classés et interprétés: la peinture rupestre et l'art japonais du XVII siècle, le cinéma américain et le cirque, l'ornement et le contrepoint musical, les différentes techniques d'acteur et la composition du sujet. Le cercle entre l'artiste, son œuvre et la perception qu'on en a, qui était envisagé dans le premier livre sur la base du conditionnement, dans le second sur celle de l'isomorphisme entre le corps, le texte et le cosmos, est rapporté, dans le troisième, à la structure de la conscience.

Dans l'écriture de ce livre, le terme «méthode» est utilisé sans ambiguïté comme une des caractéristiques essentielles du "réalisme socialiste». Il définit, d'un côté, le rapport de l'artiste à la réalité et, de l'autre, le degré de "traitement" de cette réalité dans l'œuvre d'art, qui est déterminé par le rapport à la réalité. Eisenstein conçoit sous ce terme le moyen (méthode) même de penser, c'est pourquoi son livre ne propose pas une seule formule d'analyse applicable à l'art mais une conception dynamique de la personnalité. 
Eisenstein étudie les formes de cette pensée primitive, sensible dans Totem et tabou de Freud (1913), Le Rameau d'or de James Frazer (1907-1912), La Mentalité primitive de Lucien LévyBruhl (1922, édition russe 1930), La Pensée chinoise de Marcel Granet (1934), Elemente der Völkerpsychologie. Grundlinien einer psychologischen Entwicklungsgeschichte der Menschbeit de Wilhelm Wundt (1912) et Einführung in die Entwicklungspsychologie de Heinz Werner $\left(1926^{13}\right)$, où il puise la plupart des exemples de son argumentation. Les particularités de cette pensée (indifférenciation entre "dedans» et "dehors", subjectif et objectif, microcosme et macrocosme, méthode particulière pour résoudre les conflits, figure de la pars pro toto, etc.) sont fixées dans les symboles, les rituels, les préjugés, les mythes, les contes, les œuvres d'art. L'étude de cette pensée par les psychanalystes, les ethnologues, les anthropologues, les psychologues et les linguistes est fondée sur un riche matériel empirique. Eisenstein cherche les cross connexions dynamiques entre psychologie, ethnologie, étymologie, anthropologie, esthétique et art, et il découvre les traces de cette pensée primitive dans le comportement quotidien (préjugés, réactions incontrôlées) comme dans les processus de création et de perception des ouvres d'art. Il parvient ainsi progressivement à la conviction que les particularités formalisées de la pensée sensible de type pars pro toto (réalisées par la métonymie dans la littérature ou par le gros plan au cinéma) représentent un réservoir de procédés formels. La description que les ethnographes et les psychologues donnent de cette pensée aide à reconnầtre en elle ses procédés structuraux. La tâche que se fixe Eisenstein consiste alors à découvrir comment ces procédés sont utilisés dans l'art et à transmettre ce savoir à ses collègues. Dès lors les artistes, armés de cette vision analytique qui dévoile le "comment" l'art " est fait ", pourront utiliser ce savoir dans leur pratique.

Eisenstein compare sa conception aux découvertes de Marx et de Freud, à partir d'une analogie structurelle. Freud cherche la substance de base qui explique la psyché et trouve en son fondement un conflit simple et universel. Marx opère de même avec la structure de la société. Eisenstein cherche un semblable conflit de base dans la construction de l'œuvre d'art. Il est intéressant qu'il se serve alors du même matériau analysé tantôt dans le cadre des correspon- 
dances dans Montage, tantôt dans la conception de Méthode. Ces coïncidences textuelles ont permis aux éditeurs de combiner des parties de Montage et de Méthode et de publier un texte composite comme un seul selon les circonstances: tantôt comme partie de $\mathrm{Ci}$ nématisme en français, tantôt comme partie de La Non Indifférente Nature en allemand, tantôt comme partie de Montage en russe ${ }^{14}$. Comme certains extraits de Méthode se croisent avec les «Mémoires", cela a également conduit à éditer une partie d'entre eux dans les Mémoires (éditions russe et allemande 1984, 1997, $1998^{15}$ ).

Une édition critique du livre reste donc à faire.

\section{Histoire de la création du livre. Plans d'études.}

La première ébauche de préface, la collecte du matériel, les citations recopiées, la délimitation des thèmes et des exemples commencent le 28 décembre 1932. Mais Eisenstein rapporte l'apparition de l'idée du livre à une époque plus ancienne, quand, en compagnie des psychologues Lev Vygotski et Alexandre Louria ainsi que du linguiste Nikolaï Marr ${ }^{16}$, il se penchait sur un programme de recherches consacré au langage de l'art. À ce jour, on n'a pas encore retrouvé ce projet; par ailleurs, si à partir de la deuxième moitié des années vingt, les contacts d'Eisenstein avec Louria et Vygotski furent intenses, il n'y a pas trace d'une telle proximité avec Marr.

\section{Méthode.}

Eléments de forme.

"Catalogue".

Archive.

Divers et très précieux:

1. Montage et le matériel de Taraboukine avec le dessin d'enfant ${ }^{17}$.

2. Amibe dirigée du dehors à $100 \%$ et... décors pour la "Maison des cours brisés ${ }^{18}$ ".

3. Nigrinus ${ }^{*}$ de Lucien et la vie des fourmis ${ }^{19}$.

* Mots, groupe de mots ou expression apparaissant en allemand, anglais, français ou latin dans le texte original russe. 


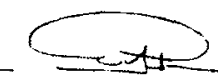

4. Zola* et Potemkin*. Einheit* [unité] du «fond» et de l'agissant, ou comment l'araignée voit ${ }^{20}$.

5. Le recul et la négation de la négation ${ }^{21}$.

6. Les tabliers et l'accouchement (Werner ${ }^{* 22}$ ).

7. Les doigts dans l'huile et la proie qui glisse des mains ${ }^{23}$.

8. Pars pro toto-Der Kneifer* [le pince-nez] ${ }^{24}$.

Et l'on reconstitue [le portrait des] voleurs à partir des traces physiognomisch $^{*}$ [physiognomoniques]. Cf. les nouveaux calculs intuit [ifs] (donner tous les termes).

9. Pensée avec la réserve de la mémoire, et non avec le rapport qu'a cette réserve avec l'application à une solution particulière.

10. Hegel. "Logique" - "Esthétique».

11. Les 24 [volumes de] Zola* renvoient à la motricité. Limage comme retour à la motricité et comment nous l'utilisons.

12. Chéréchevski $i^{25}$ et Irving ${ }^{* 26}$.

La pantomime avant la représentation. L' "avant-jeu» en général $^{27}$. Exposition en un seul acte par Pixérécourt ${ }^{* 28}$.

13. La poule de Kretschmer ${ }^{29}$ et Les Deux Orphelines ${ }^{30}$.

14. Animisme des lions sautant brusquement ${ }^{3 !}$.

15. Les dieux ${ }^{32}$. Le mode de pensée des nègres (exemples) (le processus développé à l'envers: Verdichtung ${ }^{*}$ [concentration] ${ }^{33}$.

16. Exemples de Chklovski: Natacha au théâtre ${ }^{34}$ et das konkrete Denken* [la pensée concrète].

17. Kholstomer ${ }^{35}$ et tous les totems. Les légendes. Particulièrement les Japonais. Renard fils de Patrikei ${ }^{i 36}$, etc. La bête totémique et l'ancêtre et le genre et lui-même (homme-léopard chez Storck ${ }^{* 37}$ ).

18. Versions différentes de Gogol.

"Tarass séloigna" etc* (d'après Mandelstam ${ }^{38}$ ). Spencer et la succession dans le langage ${ }^{39}$.

19. Farben "tiefe ${ }^{*}\left[\right.$ [profondeur de la couleur $\left.{ }^{40}\right]$.

20. Auflosung der Form durch Farbe [dilution de la forme par la couleur]. Picasso*.

21. Le syncrétisme, as such* [comme tel].

Rückgang* [régression] au stade de l'indifférencié.

Spécialement Mickey*, où la musique retourne à la motricité du dessin. Non seulement de façon métrique, mais mélodique aussi ${ }^{41}$.

Mais plus en détail là-dessus dans la partie sur le comique. 
22. Exemples de Gulliver. Laputa et les "objets concrets" (confronter à Natacha Rostova) ${ }^{42}$. La formation de mots chez les Clamates ${ }^{43}$ et dans le pays des chevaux (chez Swift) et leur fondation comme élément mimique qui s'est dessiné en mot selon Si-Shing ${ }^{* 44}$.

23. Montage, comme tel ${ }^{*}$. Sein Vorlaüfer ${ }^{*}$ [son prédécesseur] : la collecte des preuves dans le roman en une figure intégrale de scélérat. Excursus dans la mythologie. Isis qui collecte les parties d'Osiris. Atys. Bacchus dans l'interprétation antique la plus ancienne.

Les traits dispersés du héros, mais non pas les morceaux, sur morceaux le film et leur assemblage dans l'image globale ${ }^{45}$.

A la différence du typage morphologique, statistiquement donné à l'avance.

Observations de Quatrefage sur Syllis prolifera* — la désagrégation de vers en tas de vers indépendants (exclome [colonie] linéaire ${ }^{* 46}$ ) et Ed. Merrae ${ }^{*}$ sur le vers roulant.

Les Métazoaires devenant individus ${ }^{*}$ et Protozoa (Volvox) ${ }^{*}$ selon Girod $^{*} 335^{47}$.

Les leçons d' "Octobre ${ }^{48}$ ".

En faisant le programme:

1. Il faut faire une intervention sur scène, la prononcer

+ "Estrangement ${ }^{49}$ ".

2. Approche de la chaise sur scène et dans la vie. La logique et la dialectique. "Le bon sens". L'erreur typique des étudiants. Watson et Sherlock ${ }^{50}$. Résultat (comme la même chose fixée en forme).

3. La triade développée et enveloppée ${ }^{51}$. La développée est plus ancienne. Y a-t-il un lien avec la pensée d'autrefois, avec la prélogique?

Je me remets à Lévy-Bruhl, lu au Mexique. Werner* me tombe entre les mains.

Le sauvage, l'enfant, le schizophrène. Une thèse difficile pardonnez-moi la quantité des exemples! Et seulement ensuite les réflexions par raisonnements généraux qui confirment la vérité des mêmes thèses.

Erreurs de Lévy-Bruhl au moment de la division en logique et prélogique. Il faut trois exemples de prélog[ique], de log [ique], et de $\Delta^{52}$. La disposition par étapes, la contradiction et la relève.

1. Le prélogique et quels éléments de la $\Delta$.

Là-bas, le sujet est tout seul. 
2. La logique ét quels éléments de la $\Delta$.

Là-bas, l'objet est tout seul.

3. Dialectique.

L'unité du sub ijectif] et de l'obj [ectif].

Dialectique de l'image artistique. Double représentation par la logique et par le prélogique - enchevêtrés - de la dialectique de l'ouvre d'art. La forme par excellence* - le prélogique par la structure.

Le contenu - "particulièrement" à l'état le plus pur, i. e. par thèse ou slogan.

Euvre - unité de la forme et du contenu.

Lidée est un produit social et elle doit, pour devenir cuvre, être exprimée simultanément par la forme et par le contenu (i.e. inclure dans la clarté logique la précision du slogan et en sa langue de la pensée sensible).

Pensée sens [ible] selon Marx.

Absence de l'un ou de l'autre*.

La scolastique de La Peur ${ }^{53}$ ou du film de propagande ou de "l'art pour l'art", en partie la spontanéité de Sachko [Dovjenko]. Il captive grâce à elle, mais c'est à cause de ça quill a tort.

Le cinéma intel[lectuel], comme pierre à aiguiser, sur lequel cela a été conçu, non pas entièrement reconnu et compris selon notre cycle, souvent "figuratif": représentation figurative des notions; à noter: "les dieux» et la pen [sée] des nègres.

C'est pourquoi et l'apparence des formes et les raisons de la composition de la forme changent en principe très peu au cours de l'histoire. Leur manifestation fréquente est très diverse, car elle est liée au premier chef à une constellation sociale en changement très brutal, qui galope furieusement, tandis qu'une construction biopsychologique se meut par des changements moins brutaux.

Dans l'art "à contenu plus réduit" nous devons trouver de la manière la plus pure le tableau complet du prélogique et le maximum de conservatisme.

Le cirque:

1) Les bêtes déguisées et Marr.

2) Bisex [uality] *de clowns déguisés (de Clowns ${ }^{* 54}$ ).

3) Des animaux domestiqués (Dressur ${ }^{*}$ [dressage]).

4) Homme-serpent et hommes-oiseaux (les vols).

Le lien à l'archaïque est proche. 
En plus, les facteurs sociaux sont décisifs là-bas aussi, puisque le prélogique est une réflexion du stade indiffférencié] de la société et de ce complexe de "bêtises", comme disait Engels.

5) Les vers plats coupés en morceaux.

6) La voix d'enfant.

Les erreurs de Freud. Le sexe comme cas particulier.

Ausblick sur la Konflikttheorie* [point de vue sur la théorie des conflits].

"La lutte entre la forme et le contenu" non seulement dans la relève des étapes, mais au travers dans le processus.

Ausblick* [point de vue] dans la liquidation du conflit.

L'extase. Le pathos. Le comique. La mort.

2) Konflikttheorie* [théorie du conflit].

3) L'extase. Le pathos et le comique.

"Seul l'esprit a une histoire - dans la nature toutes les formes sont simultanées. " Hegel

Comment comprendre cela ${ }^{55}$.

On trouve en partie une explication détaillée de ces notes dans l'intervention à la réunion des travailleurs du cinéma soviétique de janvier 1935 où Eisenstein présente publiquement son projet pour la première et la dernière fois. Alors qu'il espérait triompher - dans la foulée du succès de Nikolaï Marr - , il est au contraire brutalement tourné en dérision par ses collègues (Dovjenko lui conseille d'observer les accouchements chez les femmes soviétiques plutôt que chez les polynésiennes) et n'est pas compris par ses supérieurs. Sa théorie est soumise à une critique humiliante et après l'interdiction du Pré de Béjine, on l'utilise pour expliquer la "dépravation" du film ${ }^{56}$. Néanmoins une version revue de cette conférence forme un chapitre de Méthode.

Eisenstein se prépare à présenter son projet de livre une deuxième fois dans un cycle de cours sur la psychologie de l'art à la faculté de psychologie de l'Université de Moscou. Ses notes préparatoires s'échelonnent entre 1940 et 1947 . Un résumé du cours sera publié après sa mort (en russe et en allemand ${ }^{57}$ ), mais les cours ne sont pas donnés. 
En dépit de la raillerie de ses collègues, Eisenstein poursuit son travail sur le livre. Le mot start apparaît parmi les volumineux matériaux préparatoires (315 feuillets recto verso, datés de 1933 à 1939) entre le 4 juin et le 25 juillet 1940. Eisenstein travaille dans sa maison de campagne à Kratovo. Le 25 septembre 1940 , il compose un nouveau plan, différent du premier, mais également différent du manuscrit du livre tel qu'il nous est parvenu. Il s'apprête apparemment à le publier, car le plan est émaillé de citations de Staline.

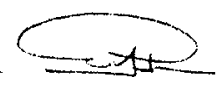

25.IX.1940.

Grundproblem $^{*}$. [le problème de fond].

All these are different levels* [tous sont des niveaux différents] : 1. Scie[ntifique] dans la forme - soc[ialiste] dans le conten $u^{58}-$ une catégorie art [istique] vide.

2. Image as interaction of $\log [\mathrm{ic}]-$ prelogic $^{*}$ [comme interaction de la logique et de la prélogique].

3. Mouvement expressif as [comme] interaction des réflexes cond[itionnés] et incond [itionnés].

4. Caractère:

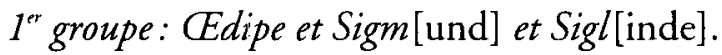

Préhist [orique] in Shift [en changement] au stade suivant (incest [ueux]).

Sur le seuil - la limite historique.

2 groupe: Hamlet et Othello (en transition: Sheylock* selon Ezra Pound: Nachklang* [écho]).

Same* [le même] : les vengeances de sang.

As far away* [comme loin] les résonances.

Confrontation avec Hamlet, l'accomplissement de la vengeance de sang (le filet!). Échange des épées là, etc.

Conflit purement shakespearien.

Dans Hamlet -- il le fallait et cela n'a pas été fait.

Dans Othello -- cela a été fait et il ne le fallait pas*.

Ensuite exemples to any caracters - caracters as such* ${ }^{*}$ [pour tout caractère - des caractères en tant que tels]. 
5) L'idéologie de membre d'un kolkhoze et les restes de l'idéologie de propriétaire là (selon Staline) dans le domaine de l'idéologie pure ${ }^{59}$.

6) La structure de l'atome.

7) Les crues et décrues.

Now... where to start from ?* [maintenant... par où commencer?].

Lidéologie.

Le caractère.

Le processus créatif et la nature de l'image.

Le mouvement expressif (de la tension dans la goutte!).

La structure de l'atome.

Et.

Les crues et décrues.

Encore aus den anderen Arbeiten.

Aber welches aus welchem ?* [des autres travaux... mais lequel vient duquel ?].

Bien sûr, commencer par: le mouvement expr[essif] et à sa droite et à sa gauche.

Le mouvement expressif - deux fonctions. D'un côté, c'est une expression de soi, de l'autre côté - une image pour la perception par les autres.

i. e. Ausdruck* [expression].

D'un côté - expression de soi - mène au caractère - idéologie.

De l'autre - à l'image pour la perception.

Le processus créatif - la nature de l'image.

La formule du réalisme soc [ialiste].

Enfin: l'homme entre l'"atome" et la "terre" - same* [le même] dans les deux notions points (plus tard).

Non, il ne faut pas de centre, mais ainsi:

1. Staline: soc [ialiste] dans la florme] et con[tenu].

2. le Staline des kolkhoziens. Idéologie. Les rapports de prod[uction] et la conscience. L'individu et la collectivité.

3. Caractère (Rückwarts* [en arrière]: Hamlet, Othello, Sheylock, Syg[mund $]+$ Sigl [inde $]^{*}$, Edipe.

4. Du caractère, i.e. du complexe d'impulsions empreint - au processus d'impulsions - au mouvement expressif.

5. Du mouvement à l'image.

6. Zurück zu Nr. 1. Stalin*. [Retour au n ${ }^{\circ} 1$ Staline $^{61}$ ]. 
Le nouveau plan insiste, à proprement parler, sur le mouvement, la représentation visuelle et le caractère, où est repérée la triade dialectique de la kinésis, la mimésis et la psyché. En même temps Eisenstein propose d'adopter un nouveau titre pour son livre: Grundproblem. Le " $M$ " raturé, qui code le manuscrit et va marquer par la suite les feuillets destinés à Méthode, apparaît le 25.IX.1941. Cependant, un tel monogramme figure déjà sur les dessins exécutés au Mexique en 1930. "M/Grundproblem" figurent comme double titre longtemps encore sur les feuillets qui ont été collectés dans les dossiers selon les thèmes désignés dans le premier plan (Natacha, Cirque, Mouvement expressif).

Eisenstein rédige alors deux manuscrits. Le premier, à Moscou en 1940 quand il a du temps pour écrire - après l'interruption de son projet "timouride", Le Canal de Fergana, en automne 1939. Il exprime son soulagement à Jay Leyda en lui écrivant qu'il aura désormais du temps pour le "livre" (sans préciser s'il s'agit de Montage ou de Méthode), après l'achèvement de la Walkyrie jusqu'à la pause de l'été 1940 - jusqu'au travail suivant dans le cinéma, qui sera Ivan le Terrible et qui lui est commandé fin janvier 1941.

Le deuxième manuscrit date de la reprise du livre au moment où, évacué en automne 1941 avec le studio de cinéma Mosfilm à Alma Ata ${ }^{62}$, le travail marque un temps d'arrêt. Le script d'Ivan le Terrible est terminé et on attend l'autorisation de tournage que Staline ne donnera qu'après la victoire de Stalingrad. Le télégramme validant le script et demandant que le film soit mis en chantier: sur-le-champ date du 14 septembre 1943. Ainsi la période de l'hiver 1942 jusqu'à l'automne 1943 se trouve être la plus fructueuse pour l'achèvement du livre dont le matériel a été réuni entre 1933 et 1941 et dont plusieurs chapitres ont déjà été ébauchés en 1940. Eisenstein rédige cette deuxième version de mémoire, ce qui, aujourd'hui, crée certaines difficultés d'édition: quelle version prendre comme base du travail d'établissement du texte, dès lors que l'on observe des différences?

De retour à Moscou, Eisenstein élabore de nouveaux chapitres, dont plusieurs ont un caractère de notes avec des "blancs". Tout le matériel de ce livre "double" peut alors être divisé de la façon suivante: d'une part, des matériaux 
préparatoires, des citations (1932-1939-1940), une première version (1940), dont certains chapitres, des variantes de l'introduction avec "les implications biographiques du problème théorique», sont écrits, ainsi que la conférence de 1935 retravaillée; d'autre part, une deuxième version (1942-1943) et la préparation de nouveaux chapitres (1944-1948).

\section{Exemples et matériaux}

Voici les notes sur le thème d'une des parties du livre.

22.1.1940. Le prélogique, la musique. Wagner et le drame. Le prélogisme langagier qui peut être observé jusqu'à l'argot contemporain et le slang d'un autre type. Dessins du livre sur likebana japonais assortis de commentaires. Le rapport entre l'art et la politique (à l'exemple de Gogol, Hegel, Wagner). Le mouvement expressif: l'Amibe, le mouvement à la surface d'une goutte, le mouvement des vagues de couleurs et de sons. Le plasma. Ébauche de biographie de Balzac. Les sociétés secrètes comme indicateur de régression. Nouveau plan d'étude (Idéologie. Caractère. Mouvement expressif. Jekyll et Hyde. Biographies: Caroll, La Fontaine, Jules Verne. Maupassant). La métonymie du sujet. (Le Malheur d'avoir de l'esprit. Les contes sur le voleur Chébarcha selon Vésélovski). L'ambivalence comme commencement inattendu dans la pensée primitive. Slang [argot]. L'ironie du slang. L'ambivalence et l'échange dans les situations du sujet (comme l'échange des vêtements entre le tsar et l'esclave; le sens, l'objet et le moyen de refléter cette opération.) (Edipe - Richard III -Dom Juan - la Matrone d'Éphèse. Recherche du père. Le trope et son incarnation dans la situation du sujet. "Et après, après avoir distingué, toucher simplement à tous les traits primitifs comme base de l'appealing* [attirance], l'anecdote dans le sujet." Lidentité de l'ornement et du rituel. Pars pro toto dans la peinture. Compositions de Degas, Menzel. Notes extraites du livre de Max Liberman sur Degas. Passage à Stendhal, notes extraites de sa biographie et $d u$ Rouge et le Noir. Répétition et rythmicité dans l'ornement. 
Barvikha, 24.VII.1940.

Trois baleines.

Qu'est-ce que la ligne? La ligne parle du mouvement.

Le philosophe chinois Van-Bi (XIr siècle?).

Le contour des dessins d'Altamira ou de Lesesain n'est pas du tout "une ligne abstraite", mais une reproduction de la toute première réaction sur le vu - la trace du mouvement sur le contour de l'objet. Il n'y pas encore de séparation du mouvement de l'oeil du mouvement de la main qui dessine.

(Cf. La réaction complexe du bébé qui vient de naître quand il réagit par la contraction de tout le corps à l'excitation de la paupière. Nos expériences avec Louria.) (cf. une telle séparation de la pensée de l'action - la pensée est mouvement et le mouvement est le contenu exclusif de la pensée. Mon état d'après-délire à Batoum en 1932.)

Ainsi, c'est une fixation de celui qui se fait remarquer le premier du mouvement (cf. Engels: d'abord le mouvement, et ensuite ce qui bouge!).

Le mouvement de l'objet lui-même ou le mouvement de l'cil autour du contour de l'objet n'ont pas encore de différence de principe! (subjectif $=$ objectif!) (N.B. À l'origine le même que sur le sommet. Cf. l'absence de différence de principe dans la méthode de composition du cadre de traces d'objets en mouvement ou de formes immobiles!)

De la même façon la précision du contour n'est qu'enregistrement d'un trait de l'objet, du plus frappant - du mouvement.

Et sur les "sominets" la même révélation suprême de la synthèse du phénomène souvre quand elle est comprise comme mouvement! (Plékhanov: tout phénomène en fin de compte se réduit au mouvement!) Et la synthèse suprême d'un point de vue figuratif est donnée aussi... par le trait (ou par le schéma pour les notions, les organisations, les systèmes, etc.)

Ce stade est "en dehors de l'art" (Nordische Kunst ${ }^{* 63}$ en parle bien).

Il appartient à l'étape de la pensée précédente même par rapport au complexe et au paralogique: au stade de la réflexion de conscience au niveau de la réflexion par la surface de l'eau.

(Se renseigner et réfléchir sur le stade précomplexe de la pensée.) 
La "géométrisation" commence par les premières poussées de la pensée complexe. "Apparition" de la pars pro toto qui n'existait pas avant. Et du tableau général du mouvement-ébauche, il suffit d'un élément - encore une fois, apparemment la caractéristique du mouvement de l'ail sur l'objet. La "verticalité", la "rondeur", l' "étendue", etc. Graphiquement composés des lignes les plus simples, et selon leur contenu - caractéristiques mobiles de l'ail par rapport à l'objet.

Parce que le trait, comme la ligne, non pas dans notre interprétation, mais en tant que trace de mouvement, est la pars pro toto de tout l'objet, par sa propriété la plus frappante - sa mobilité.

Ici coïncidence absolue avec le phénomène sonore primitif, cf. Cushing. Manual concepts*, comment les mots se forment du son de l'acte physique (mater*).

La ressemblance totale avec la notion de Lautbild* [image sonore]. Car l'ail dessiné comme un cercle n'est pas une "schématisation" de l'ceil mais le tracé de l'cil observateur sur le contour de l'ail représenté (ou le contour du visage, ou la forme du sein, etc.)

Sans intention, il sapproche des traits "caractéristiques", non pas à travers l'analyse, mais à force de frapper. Car c'est ainsi que le surnom apparaît, lui aussi, selon sa propriété la plus frappante avant tout*.

Mais nous disons "homme carré", "petite sphère", etc. (cf. Tolstoï, Pierre Bézoukhov pour Natacha - le "carré bleu").

Et c'est ainsi que le "sauvage" peint.

[...]

Barvikha, 20.VII.1940.

Not to be forgotten * [à ne pas oublier]:

Hogarth: ("Analysis of beauty")*

Sur l'intérêt de la ligne comme vestige... de la vie errante des chasseurs, le plaisir de la poursuivre, la suivre, courir après elle.

Ajouter quelque chose sur la fugue — de la racine "flight" fuite! i. e. le même élément dans le son dans la forme haute. Mais le même dans la musique — dans la mélodie — "we don't hear a melody - we can or cannot follow it" [nous n'entendons pas une mélodie, nous la suivons ou non] (Lang)*.

Impact du contour fermé.

Le contour fermé concentre dans l'action le "corps de superficie" qu'il renferme. Et le mouvement embrasse non seulement la ligne qui 


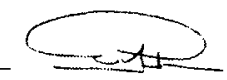

"court", cette superficie suivant le contour, mais fait "trembler» en mouvement la zone embrassée. Un tel dessin ressemble dynamiquement au mouvement des cellules d'amibes à l'aide de pseudopodes:
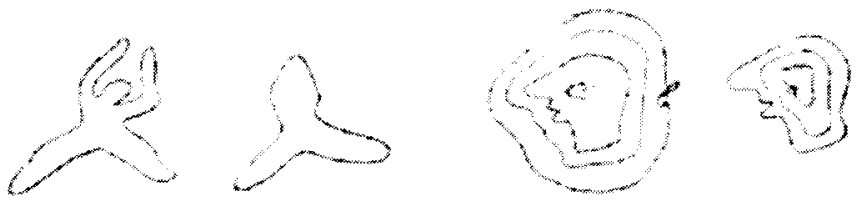

i. e. par le mouvement non seulement (et pas exclusivement) $d u$ contour, comme la course de la superficie qui reluit de forme en forme.

Il faut réaliser que ces reluisances sont un tableau parfaitement concret.

Car une telle figure fermée a des pôles d'orientation vers les figures géométriques les plus élémentaires comme exprimant séparément la tendance (ce que le géométrisme du dessin est au premier stade, quand la chose est remplacée, par ex [emple] «la ligne de son orientation principale").

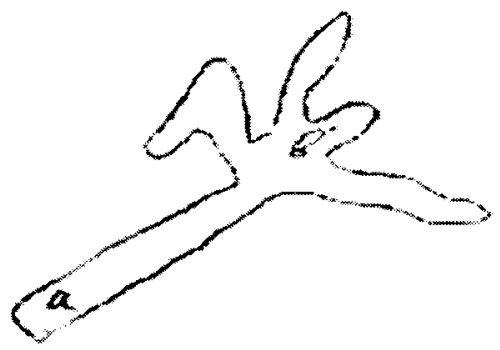

Entre ces pôles (a) et (b) se joue le jeu de la superficie (c).

Ou autrement encore, si la figure est complexe, alors sur éléments, ou encore il y a comme un désir de "faire entrer" tout (b) dans (a) ou tout (a) dans (b).

Quand le dessin n'est pas fermé, ce facteur est partiel ou complètement absent: il y a moins de jeu du mouvement des contractions sur la superficie, moins de systoles-dyastoles de superficie vit. [ale]. 
N.B. : d'où le passage au chatoiement des volumes et des masses les uns dans les autres, mais aussi les espaces illuminés et obscurs les uns à travers les autres. La couleur - entre les deux.

Barvikha, 3.VII.1940.

Trois baleines.

Je pense que "mon mérite le plus grand" dans la question du géométrisme du dessin primitif, c'est la séparation du géométrisme de l'ornement. I. e. qu'il n'y a pas un phénomène, mais deux. La répétitivité dans l'ornement, je la réduis à la "troisième baleine" - à la répétitivité as such ${ }^{*}$, en tant qu'un phénomène indépendant, proche d'un processus organique — nécessairement rythmique. (cf. Kretschmer, Mediz[inishe] Psychologie ${ }^{*}$ ).

Le géométrisme - du complètement indépendant: comme un nucleus* intellectuel - où la pars pro toto* se déplace déjà d'un trait de l'objet $\underline{a}$ sa propriété à sa qualité (étendue, orientation, etc. ${ }^{*}$ ) en remplaçant par le toto qui est déjà une propriété, pas simplement une qualité.

Le géométrisme de l'ornement dans ce cas est une synthèse primitive où l'intellectualisme pur de ce stade — le géométrisme - est uni à la sensibilité maximum - régressive même pour le prélogique! à la répétitivité comme élément de phénomène purement physiologique (i. e. encore plus bas que le prélogique). Voilà comment il faut comprendre l'ornement. Et là le deuxième mérite: après la séparation - l'unification dans une synthèse!

$[\ldots]$

Le géométrisme naît au moment de la création des outils, i. e. au moment de la séparation primitive de la propriété du porteur de propriété (car le géométrisme est complètement dasselbe * [le même] !). Car c'est de ce moment que naît le sommet des possibilités de fonctionnement de la pens[ée].

Ici même l'abandon de l'automarquage (tatoo ${ }^{*}$ à la coloration des objets environnants. Regress* : les criminels se tatouent.

Le géométrisme, c'est le même stade dans le dessin - déjà pas simplement n'importe quelle partie, prise souvent par hasard, mais une propriété isolable (orientation, orientation mutuelle, contour, etc.) - le premier travail d'abstraction.

Et quand même cette conscience ne sait pas encore choisir les unités caractér[istiques] principales de ces propriétés et à l'intérieur de chacune 


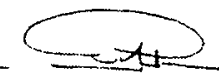

de ces propriétés, c'est pourquoi, par exemple, nous "ne reconnaissons pas» dans les dessins stylisés, en particulier dans les plus primitifs, ce quils représentent exactement (ils ne figurent pas avec exactitude).

Voici un exemple de texte d'une des dernières ébauches.

7.1.1948. "Les baigneuses» de Degas.

Degas - Gogol.

Extraordinairement important par ses conséquences. Noter: deux moyens dans la composition du cadre: le remplissage des limites et le découpage de ce qui se passe devant la caméra. Ramper en soi-même - tourné à l'intérieur - dans le caractère de Degas pour l'importance des cadres englobant dans ses tableaux. À proprement parler, c'est le plus important de son excellence et sa particularité.

Loriginalité de Degas.

La différence entre Degas et Gogol réside dans ceci que chez Gogol, la $M L B^{* 64}[\mathrm{part}]$ dans l'ordre imago-symbolique et est sousentendue dans les objets de la figuration. Le "cadre" englobant de Degas - chez Gogol est sous les espèces du... manteau "enveloppant", de la calèche embrassante (et la "troïka-oiseau", et la calèche et la passion de Gogol de voyager en calèche, etc.).

Chez Degas - le principe du procédé, chez Gogol - l'objet ambigu (le manteau chaud, etc., la $M L B^{*}$, etc ${ }^{*}$ ).

A noter*: la position du détail au premier plan et le principe du super-individualisme. (A proprement parler, cela aussi se réduit à la $M L B^{*}$ - car cela représente la projection de l'égocentrisme, puisque la situation de la $M L B^{*}$ est égoïste et égocentriste au maximum.)

Nietzsche dans ce sens.

Degas en ce sens. (De côté) - El Greco et Piranèse.

Nietzsche - Degas - Gogol.

La vie amoureuse et un certain surhommisme avec un égocentrisme très souligné chez tous les trois (chez moi aussi : c'est pourquoi je vois ६̧a). 
Jusqu’à quel degré la $M L B^{*}$ chez Degas est réellement Umgränzung* [délimitation] dans le découpage, en principe typique chez lui, du "cadre", se manifeste à partir de l'exemple suivant.

Celui de (ma favorite). La ferme qui se baigne.

Ici est remarquable le caractère itératif de l'Umgränzung* [délimitation], la pluralité des cadres: en plus, le cadre triple (quadruple) - dans les trois cas de qualité différente.

A partir du cadrage de l'objet (par le tub).

Par le mixte (le cadre + les détails à l'intérieur du tableau: le couvercle de la cuvette ou la banquette avec le broc) au purement abstrait, i. e. au découpage - au cadrage de limage par le cadre.

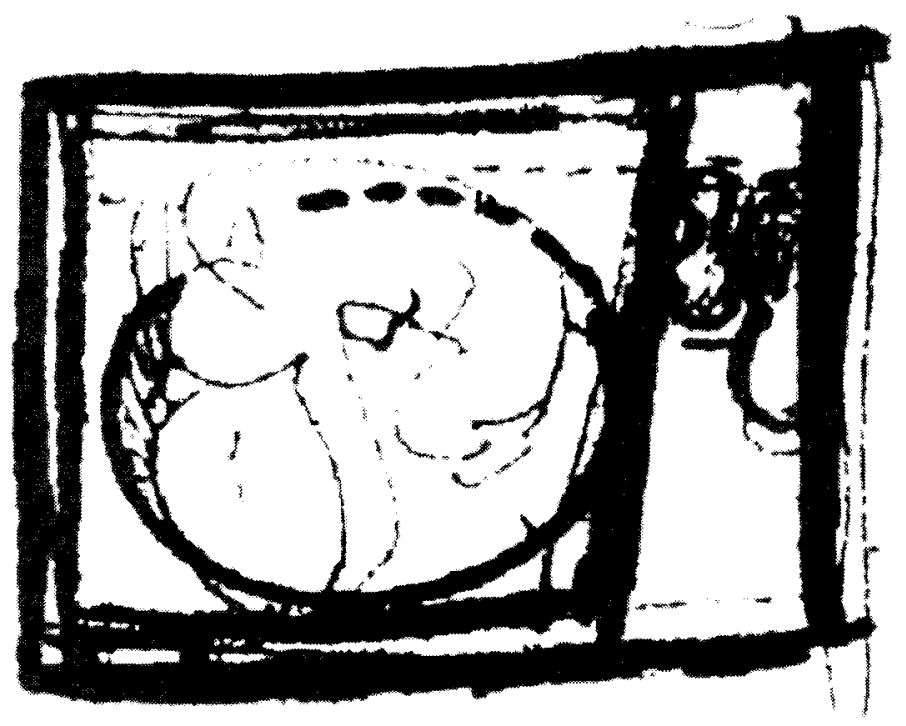

Le contour:

- objectif;

- mixte;

- cadré.

A noter ${ }^{*}$ : le caractère recherché des contours consiste encore, par exemple, dans ce que le cercle total (du tub) sachève par la ligne du dos...

Le deuxième cadrage est, au sens strict, bâti au-dessus non pas par le cadre non plus, mais par la ligne de lintersection du plancher et d'un petit morceau de mur... I. e. il donne un contour graphique à 
la jointure de deux constructions de plans dissociés dans l'espace: la ligne du boral de la cuvette - de la banquette et la ligne de l'intersection du plancher avec le mur. (Très rafinné* [sic]!)

Encore très remarquable: le mouvement du cercle au rectangle.

I. e. dans cette tablette seule s'est imprimé le processus de transformation du cercle Womb* [matrice] au rectangle dwelling place ${ }^{*}$ [lieu de séjour] (et tout ce qui a été dit de... la "quadrature du cercle" des mathématiques, comme reflet de la même sphère urgeplazma*-tique sur la superficie osseuse.

Encore et dans Schleife ${ }^{*}$ [le noud] et la raideur de l'écriture (la reproduction dans Propyläen Kunstgeschichte, "Realismus und Impressionismus", p. 477).

La même observation découvre aussi un sens implicite de mon analyse de la "Ballerine" comme système de cercles concentriques décalés.

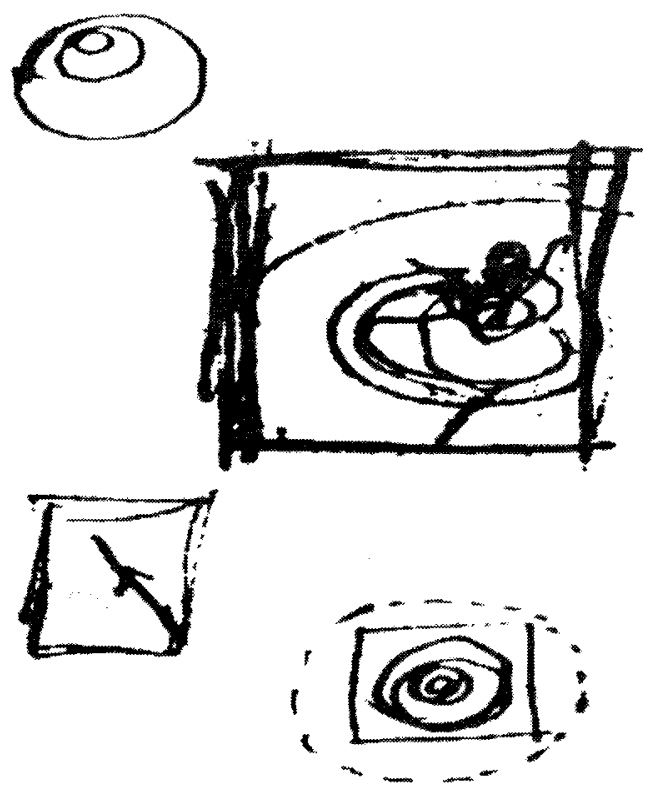

Le déplacement des cercles concentriques résout le thème de l'" absorbement" du spectateur non représenté dans l'image.

I. e. l'interaction de la flèche entrainante et du système par les cercles concentriques normaux. 
(Un tel entraînement du "bors-cadre" pour Degas dans sa position générale sur la pars pro toto est remarqué encore par Tugendhold, qui parle mal de la pars pro toto $)$. Cf. principalement George Rivière. "Mr. Degas", Florenz, 1935, p. 67*): Ce que disait Degas du réalisme, si l'on en croit la plume de son ami Duranty... "Par le dos, comme il nous apparaît, je dévoile le tempérament, l'âge, la position sociale; dans la paire de mains d'un musicien, nous devons exprimer le caractère de musicien, de marchand; dans le geste tout un enchaînement de sentiments. ")

Cela renforce la base de limage des cercles concentriques (Cf. Gogol: le cercle rose et "dans son ventre joua le nourrisson").

Leur pré-image dans la MLB*.

À côté de ça, c'est Sérov qui est remarquable.

Le portrait d'Ermolova par excellence*, où le même procédé sous l'espèce de rectangles concentriques (des étapes de cercle!).

La base sous-jacente du principe des cadres concentriques est apparemment la même (à noter* la communauté de caractère de Sérov et de Degas!!! Sérov a aussi du caractère - Dieu sait lequel! introvers $^{*}$ aussi.)

Ce qui est remarquable, n'est pas tant (et non seulement) la présence de la formule de départ, son "identité" chez les deux, mais, principalement: son utilisation en vue d'une application à ses tâches spécifiques.

La chaîne réfléchie de "cadres" et sa lecture dans le portrait d'Ermolova (comme "prosternation", etc. ${ }^{65}$ ) et chez Degas comme lasso* qui entraîne "le spectateur hors cadre". (Cf. Rodin* sur son "Balzac" - "Je veux qu'on lise autour de lui, la chambre, le désordre, les manuscrits", etc ${ }^{*}$.) Moins raffiné que chez Sérov, - et probablement parce qu'elle est là - la solution est dramatique et sujétale, et non comme dans "Ermolova" — le rapport avant tout* à la figure, la solution sur le comportement n'étant pas de dramaturgie ni de sujet (tout le rapport est exprimé par les moyens de la composition), c'est Menchikov à Bérezovo de Sourikov.

C'est encore Ioffé qui dans son "Histoire synthétique de l'Antiquité "le lit comme une composition concentrique en "cercles" (fig. de rectangle) qui répète le détail central - le poing serré de Menchikov.

Cf. "Histoire synthétique de l'art", éd. 1933, p. 309: 
"Les figures sunissent dans un tout complet, comme un cercle fermé dont le centre est le poing serré de Menchikov, le coupable et la victime du bagne..."

J'énumérerai ici le système des rectangles concentriques.

Le poing.

L'anneau de la famille.

L'anneau de la tête de la fenêtre... du lutrin.

L'anneau du cadrage par le contour du tableau.

C'est caractéristique de Sourikov: ce "système" n'est pas tiré du fonds d'une obsession* personnelle par l'image de cercles concentriques adaptés au but (Degas, Sérov), mais ce schéma est choisi comme convenant le mieux dans la situation figurée. Comme dans un autre cas - la fameuse "diagonale" de Morozova". La longue recherche du schéma qui convient le mieux. L'arrivée au schéma est ici très distincte. Probablement, est-ce là encore un des indices du fait que la magie est absente chez Sourikov à la différence des deux autres: Sérov et Degas!

\section{Problèmes d'édition}

Non seulement l'identification des textes appartenant à $M e^{\prime}$ thode et leur attribution posent-elles un problème à tout éditeur de ce livre, mais encore en est-il ainsi de la structure même de l'édition. Il convient d'opter pour un principe de composition, et cette décision sera déterminée non seulement par les traits du texte eisensteinien "brut", mais aussi par la manière dont l'éditeur comprend le texte lui-même: encyclopédie, autobiographie, journal, manuscrit de livre sur la théorie de l'art ou notes préparatoires pour celle-ci. La définition du genre auquel appartient ce livre résout des questions de publication.

La commission exécutive près l'Union des cinéastes que présidait Sergueï Ioutkevitch, et dont faisaient partie Rostislav Iouréniev, Semion Freilikh, Naoum Kleiman et d'autres, avait préparé pour l'édition une partie du manuscrit en transformant les fragments en un texte fluide (de tels passages existent, cela va de soi, chez Eisenstein), et en rejetant complètement ce qui était inachevé et resté à l'état de notes. Même dans cet état châtié 
cependant le livre demeurait impubliable. C'est à cette version quappartiennent, en général, les extraits publiés depuis quelques années, donnant l'impression d'un livre achevé, continu. Or le texte consiste en une série de fragments, compris comme un tout, renvoyant à la conception du fragment dans le romantisme telle que l'a renouvelée l'art $\mathrm{du} \mathrm{Xx}^{\mathrm{e}}$ siècle.

Eisenstein pose le fragment comme encyclopédie; il évoque cela dans son premier point de départ avec Lucien (que Bakhtine défnit comme un encyclopédiste). Mais si l'on parle d'une encyclopédie de ce type, n'oublions pas qu'il s'agit, chez Lucien, d'une fiction, d'un texte littéraire qui se donne comme allégorie d'encyclopédie (comme Ulysse). Ce n'est pas par hasard que, dans son manuscrit, Eisenstein mentionne les Védas qui présentent une description métaphorique de la genèse du monde - et une théorie chiffrée de la science. La clef de la double lecture des Védas n'était donnée qu'aux prêtres initiés au savoir spécifique. La clef de lecture de la métaphore littéraire est donnée le plus souvent par l'auteur (Joyce-Gilbert). Or Eisenstein ne nous donne pas la clef qui aiderait à comprendre la composition de Méthode.

Tout le texte peut être considéré comme un commentaire de son plan dessiné en pointillé où les proportions entre le corps du texte et les marges sont déplacées. Voilà qui peut aussi déterminer la forme de la publication où l'éditeur/le commentateur comble les lacunes par les citations retrouvées, des sources indiquées ou non, déplaçant les proportions entre le texte de l'auteur et ses suppléments. Montage ressemble à un énorme recueil, un collage de citations et de matériaux préparatoires, comparable au Passagenwerk de Walter Benjamin. Mais si Benjamin pouvait imaginer que son livre devienne, dans l'idéal, un montage de citations d'autres que lui, des commentaires (comme il l'avoua à Adorno), avec Eisenstein il n'en va pas de même. Le recueil de ses citations provient de sources qui s'excluent souvent l'une l'autre, de disciplines qui se nient (comme la psychologie et la psychanalyse), pour lesquelles Eisenstein crée un nouveau contexte et de nouvelles références, en les recodant parfois totalement.

En même temps, Méthode est un livre "thérapeutique" comme l'autobiographie. Les toutes premières notes de My Art 
in Life renvoient aux tentatives de se débarrasser de sa névrose, de sa dépression et de ses doutes. Le lien entre l'autobiographie et l'auto-analyse thérapeutique est enraciné dans des traditions lointaines. Mais il semble que personne n'ait élaboré une théorie en tant qu'analyse thérapeutique. Mikhail lampolski, qui considère la "théorie" eisensteinienne "comme citation", propose pourtant une telle explication (1999, p. 58):

\begin{abstract}
Tout commence par ses problèmes intimes propres et retourne à ses problèmes. Le théoricien lui-même joue le rôle du "tiers", du "facteur ultime", de l'ego transcendantal. Son ego est un être qui reflète toutes les notions théoriques, crée les oppositions et les réconcilie pour en faire des similitudes. Il opère une grande synthèse. Les fragments théoriques, les "citations", comme je les appelle, sont ainsi réellement assimilés par Eisenstein et transformés en symptômes de ses propres clysfonctionnements. Il souffre d'une maladie de la volonté qui se manifeste dans ses inhibitions sexuelles, mais la volonté elle-même est un produit des inhibitions en termes réflexologiques. Trouver une similitude profonde entre le libre arbitre et l'automatisme réflexologique n'est pas une simple tâche théorique, mais une nécessité thérapeutique. Le projet de théorisation dans son ensemble est guidé par la maladie d'Eisenstein. Et réglementé par les similitudes et dissimilitudes enracinées dans sa propre expérience personnelle.
\end{abstract}

Mais est-ce de la théorie? Le livre frappe par le didactisme de son idée centrale, dont Eisenstein tente de montrer l'universalité, et par la plurivocalité des langues, le montage des citations et du matériel "des autres" qui est mis en forme par le contexte comme "le sien propre». Le plus simple est de prendre ce texte comme un "Journal». Dostoïevski a écrit son Journal d'écrivain comme une ceuvre limite entre fiction et document. Le texte d'Eisenstein est placé sur la frontière entre le journal intime, l'auto-analyse, les notes de lecture (incluant livres, journaux, revues d'orientations les plus diverses), les dessins et la théorie. Le dessin est inclus dans le texte pour mieux comprendre les constructions analytiques grâce à la visualisation. Le journal intime sous-entend l'inachèvement et l'intime. «Pacha est maintenant 
entrée avec le déjeuner, et le déjeuner a laissé une tache sur le manuscrit. "Une trace. Après la tache (ou le déjeuner ?), la pensée de l'auteur se développe dans une autre direction. Ce sont de tels passages que Naoum Kleiman a retranchés de la publication de Disney, de même que tous les morceaux autobiographiques thérapeutiques concernant les rapports avec les femmes et les hommes.

\section{lrédit}

Alma Ata. Montagne. Bungalow. Le 2. XII.1943

As usual* [comme d'habitude]. Il suffit, en suffocant d'admiration, de lire quelque chose, quil se trouve en plus que cela hits* [frappe] absolument quelque part dans les matériaux de recherche. C'est très compréhensible. Thrilling* [excitantes] sont pour moi les choses d'un genre très particulier... elles mintéressent du point de vue de chercheur. Il est clair cependant que this kind of stuff* [ce genre de choses] entre chez moi d'habitude par l'amour*. That gives a certain suavity which emphasizes* au lieu de l'ennui académique something youthful, not to say boyish! Anyhow* [Cela donne une certaine suavité qui accentue... quelque chose de jeune, pour ne pas dire de gamin! En tout cas], emballé par St. Mawr de D. Lawrence ${ }^{67}$. Alors, emballé par St. Mawr, cet "hymne" to the Great God Pan* [au grand Dieu Pan] qui est maintenant devenu rien de plus que Great Goat Pan [le grand Bouc Pan] (je cite l'auteur* the puss about Pan may not be of how high a class - but fitting its purpose ${ }^{*}$ [le bruit sur Pan peut ne pas être d'une classe élevée -, mais il remplit son objectif]. Quelque part in the background lures ${ }^{*}$ [sur le fond brouillé]— reminiscence.

Agnia. La période du Proletkult. Le Sage.

Horseback riding for all of $u s^{*}$ [monter à cheval pour nous tous] Agnia. With this Dostoieusky name* [avec ce nom de chez Dostoievski ${ }^{68}$ ].

Agnia. The first I slept with* [la première avec qui j'ai couché] Agnia. Weaving into the pattern*. Arvatov ${ }^{69}$. Vertov. Myself and a big big... white stallion* [tissant dans le modèle. Arvatov. Vertov. Moi-même et un grand, grand... étalon blanc] (au manège, passage Granatovy). 


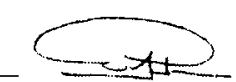

L'intimité* entre Agnia et l'étalon (blanc) était évidente.

Le rival s'efforģait d'engloutir (?) ses petites mains dans ses lèvres chaudes.

(Bizarre, je me souviens qu'il m'était absolument indifférent que la main soit petite.)

Arvatov est devenu fou.

Principalement, à cause de moi.

L'étalon n'y était pour rien.

Plusieurs années après.

Le destin l'a voulu.

Agnia habite (habitait) dans le même immeuble — l'aile de la maison de Hertzen - avec Madame ${ }^{70}$. Mariée avec un national.

L'enfant avec les yeux bridés et les pommettes saillantes.

Sont devenues amies avec Madame, certainement, sur le thème de moi.

L'étalon blanc était bien.

Pitié pour Arvatov.

Agnia sempoisonnait.

Je suis parti avec Véra lanoukova ${ }^{71}$.

Pourtant tout sauflétalon n'a aucun rapport là.

St. Mawr fait un petit chapitre dans mon Disney ${ }^{72}$.

Totémiquement, l'épos bestial de Lawrence domesticated and longing $^{*}$ (apparemment still the peace par excellence* as counterpart to ${ }^{*}$ Lautréamont et son épos activement sadique de la bête as opposed $^{*}$ à la connexion bestiale de Lawrence) [Lawrence domestiqué et désirant (apparemment toujours la paix par excellence comme contrepartie à Lautréamont et son épos... de la bête comme opposé à...)].

À noter* en plus:

Etalon et feu.

La démarche très intéressante de la métaphore non seulement de l'homme à travers la bête (et vice versa), mais la bête à travers la bête, et par là son humanisation * bizarre:

St. Mawr saute comme un poisson, est assis, les jambes sur les côtés, comme un lézard. Les chevaux glissent au-dessus des champs comme les... papillons en train de jouer. (A noter* : le mariage de Lou et Rico est comparé au même jeu des papillons. - N.B. totalement sans rapport et absolument disjoint de ce troupeau). 


\section{Inédit}

Cela fait penser au Cirque sous l'eau de Disney avec la métamorphose des bêtes l'une dans l'autre. The elephant faming* d'un groupe d'octopodes, le poisson-tigre dans la cage, [illisible] la marche sur les chevaux de mer, etc. ${ }^{73}$

Qui voudrait lire et qui voudrait publier un tel livre?

L'édition anglaise de l'opus de Walter Benjamin, vingt ans après l'édition allemande, a engendré des réactions qui sont loin d'être enthousiastes ${ }^{74}$. Il est possible que l'édition de l'opus magnum d'Eisenstein engendre, elle aussi, une certaine incompréhension. L'irruption permanente de l'intime, non destiné à la publication, l'allégeance à des usages de convention (citations de Staline), l'ironie, l'autoglorification (commentaire fréquent à propos de ses pensées: "Great!» [Magnifique]), les indécences, et l'apologétique créent un portrait d'Eisenstein assez inattendu. Et comme le nouveau discours dominant qui prendrait la relève $\mathrm{du}$ "déconstructionnisme» ne se dessine pas, ce portrait est difficile à "utiliser». Jusqu'à aujourd'hui en effet, les éditions d'Eisenstein ont appréhendé la dynamique de sa personnalité et de son œuvre en fonction des modes intellectuelles. Le premier élan de publication remonte aux années soixante, il s'agissait alors d'extraits des mémoires qui se situaient d'une part dans la vague de la littérature soviétique du "Dégel " au cours desquelles on réhabilita l'avant-garde et des artistes dont la référence avait disparu pour le public depuis trente ans, d'autre part dans le courant de l'autoréflexivité narcissique des autobiographies existentialistes à la Sartre (Les Mots). Dans les années soixante-dix, lorsqu'on crut que le structuralisme allait sauver les sciences humaines au nom de l'objectivité, on a lu Eisenstein comme un structuraliste de gauche et un proto-sémioticien, ce qui a assuré l'intérêt pour son ouvre en URSS comme en Occident. Les travaux consacrés à Eisenstein à cette époque se situent au croisement du marxisme et du structuralisme. Dans les années quatrevingt, les Français, les Allemands et les Italiens ont découvert en Eisenstein un nouveau théoricien, qui allait de la structure à la psychanalyse, en accord avec les nouveaux discours ayant 
supplanté le structuralisme. Le privilège accordé à ses analyses picturales ou architecturales s'est ensuite placé dans une nouvelle grille analytique axée autour de la problématique du regard et du positionnement du corps dans l'espace. Reçu avec retard en Russie, Michel Foucault a enfin recentré l'intérêt sur le corps, la délégation de pouvoir et la sexualité. On cherche désormais dans son "Journal" de quoi fournir la matière d'une nouvelle approche, mais il n'est toujours pas question de le publier; d'ailleurs les «journaux » restèrent inaccessibles jusqu’à très récemment. Est-ce parce qu'on craint que l'Eisenstein "structuraliste-de-gauche" et victime du "système totalitaire" (dans le paradigme des années soixante), l'Eisenstein culturologue et attaché à la question du regard et de la perspective plurifocale (dans le paradigme des années quatre-vingt) disparaisse derrière la figure d'un esthète homosexuel corrompu par le pouvoir?

Quoi qu'il en soit, la maison d'édition berlinoise PotemkinPress tente depuis deux ans déjà de préparer l'édition de $M \dot{e}-$ thode, sans avoir malheureusement pu obtenir, pour diverses raisons, la copie complète du manuscrit de la part des archives de Russie (RGALI), qui ne manifestent guère d'intérêt pour une telle publication.

Face à la situation de ces inédits, il n'en est que plus étrange de voir Naoum Kleiman édicter de nouveaux principes censés réglementer l'accès aux archives dans un entretien avec Oleg Aronson pour iskousstvo kino:

Aronson : - À côté de la censure officielle possible, vous avez évoqué à plusieurs reprises, dans la conversation, le thème de la censure ad personam. Il est dès lors très probable que vous ne vouliez pas que telles ou telles choses soient publiées par crainte de leur interprétation... Kleiman: - Ce n'est pas de la censure. Distinguons la censure comme déformation violente qui exerce une pression et la censure au sens du "tact». Quand quelqu'un regarde par un trou de serrure, il ne s'agit pas de censure. C'est une question de tact.

A. : - C'est très subjectif. Comment distinguer un chercheur "qui a du tact " d'un "voyeur"?

K.: - Je ne me place pas au-dessus des chercheurs. Je ne crois pas qu'il faille fermer l'accès à quoi que ce soit, 
y compris le domaine érotique. Mais une publication exige du tact. Je suis pour un conseil de personnes compétentes et responsables qui établisse où commence "le trou de serrure".

A. : - Cela signifie que les publications devraient recevoir la permission de ce conseil?

K. : - On peut le dire ainsi. Ce procédé est en vigueur dans le monde entier et n'est pas du tout une invention du pouvoir soviétique.

A. : - Nous savons que les uns accusent Eisenstein de stalinisme, d'autres l'interprètent vulgairement, d'autres encore ne l'aiment simplement pas... Et en ce cas vous, par exemple, ou d'autres membres du conseil probablement, en éprouvant le sentiment personnel d'avoir affaire à un manque de tact, que vous le vouliez ou non, vous allez exercer une certaine censure psychologiquel ${ }^{75}$.

L'état de l'édition des textes théoriques d'Eisenstein est un problème d'autant plus grave que lui-même considérait sa recherche théorique comme principale au sein de son activité:

Tout ce que nous faisons çà et là, ou n'importe où, écrit-il, de Hollywood, le 3 septembre 1930 à Péra Attachéva, n'est qu'un haut-parleur pour qu'on lise ensuite le livre ${ }^{76}$.

Ses films mêmes sont vus comme la "promotion" de sa théorie.

\section{Traduction du russe: Egor Ovtcharenko}

\section{NOTES}

1. Viatcheslav Ivanov, Essais sur l'histoire de la sémiotique en URSS (Moscou: Naouka, 1977). Le philosophe Valéri Podoroga prépare un ouvrage à paraître aux éditions Logos à Moscou.

2. "Disney" dans: Alexandre Prokhorov et Boris Raushenbach (direction), Problemy sinteza v khudozhestvennoj kul'ture [Problèmes de synthèse dans la culture artistique] (Moscou, 1985, p. 209-284). En anglais: Eisenstein, On Disney (Calcutta: Seagull Books, 1986 ; Jay Leyda, direction). En français: Eisenstein, Walt Disney (Strasbourg: Circé, 1991 ; Naoum Kleiman, direction). “O detektive» dans: Prikljuchencheskij fil'm. Puti i poiski (Moscou: VNIIK, 1980, p. 134-160; Alexandre 
Trochine, direction). En anglais : Eisenstein, The Psychology of Composition (Calcutta : Seagull Books, 1987, p. 57-82; Jay Leyda et Alan Upchurch, direction). "Rodin et Rilke» dans: Cinématisme (Bruxelles: Complexe, 1980; François Albera, direction). En russe dans: Kinovedtcheskie zapiski, n" 34, 1996.

3. À l'inverse, des textes comme "Pair et impair" et "Le dédoublement de l'unique" - d'abord publiés en traduction française dans Cinématisme - ont été édités par Naoum Kleiman comme faisant partie de Montage, en dépit de leur proximité avec $M \dot{\ell}$ thode. Ils l'ont été à partir du manuscrit rattaché à Méthode (op. 2, ed. khr. 261, 262, 263) et à Montage (op. 2, ed. 316) avec omission d'une partie des deux manuscrits: le texte "Rodin et Rilke", rattaché à Méthode lors de sa publication russe en 1996, est pourtant, lui, plutôt proche de Montage. Eisenstein lui-même rapportait ses analyses à un livre comme à l'autre en les complétant et les corrigeant à l'intérieur de différents manuscrits. Aussi peut-on les éditer à la fois dans l'un et l'autre livre, car leur lecture, au sein des conceptions diamétralement opposées de Montage et de Méthode, en déplace l'accent. L'attribution de "Disney" à Méthode n'est pas attestée dans le manuscrit tel qu'il est annoté, mais peut être trouvée dans le plan de l'édition de 1933, voir infra.

4. Voir son livre Kino segodnya. Vertov, Koulechov, Eisenstein (Moscou: Teakinopetchat, 1924).

5. Boris Eikhenbiaum (direction), Poètika kino (Moscou-Leningrad: Akademia, 1927); en français: François Albera (direction), Les formalistes russes et le cinéma (Paris: Nathan-Université, 1996); Vsevolod Poudovkine, Kinorezhisseur i kinomaterial (Moscou: Kinopetchat, 1926); en anglais: Film technique: Five Essays and two Addresses, translated and annotated by Ivor Montagu (London: G. Newnes, 1935); Semion Timochenko, Iskousstvo kino. Montazh filma (Leningrad: Academia, 1926); en allemand "Filmkunst und Filmschnitt", en annexe à W. Pudowkin, Filmregie und Filmmanuskript (Berlin: Verlag der Lichtbildbuhne, 1928); Lev Koulechov, Iskousstvo kino - moi opyt (Moscou-Leningrad: Teakinopetchat, 1929); en français: L. Kouléchov, L'Art du cinéma et autres écrits 1917-1934 (Lausanne: LÂge d'Homme, 1994).

6. RGALI f. 1923, op. 1, ed. chr. 1030. En allemand dans: S. Eisenstein, Das dynamische Quadrat. Schriften zum Film (Leipzig: Reklam, 1988, p. 344); en français dans: François Albera, Eisenstein et le constructivisme (Lausanne: L'Âge d'Homme, 1990, p. 17).

7. Voir O. Bulgakova, Sergej Eisenstein: drei Utopien. Architektentwürfe zur Filmtheorie (Berlin: PotemkinPress, 1996, p. 31-108).

8. "A Statement * (Close up, vol. III, n" 4, London-Territet, October 1928, p. 1013). "The New Language of Cinematography" (Close up, vol. IV, n" 5, May 1929, p. 10-13).

9. Sur cette correspondance et le projet de livre, voir François Albera, Eisenstein et le constructivisme (op. cit., p. 17-21).

10. Projet publié par Naoum Kleiman (Kinovedtcheskie zapiski, $\mathrm{n}^{\text {os }} 36 / 37,1998$, p.13-23).

11. Stuart Gilbert, James Joyce's Ulysses. A Study (New York: Alfred A. Knopf, 1930).

12. Otto Rank, Art and Artist (New York: Tudor 1932, p. 120, cité par J.H. Philips, dans Psychoanalyse und Symbolik, Bern und Stuttgart: Verlag Hans Huber, 1962, p. 127).

13. Heinz Werner, Einfuibrung in die Entwicklungspsychologie (Leipzig: I. A. Barth, 1926).

14. Voir note 2.

15. Dans la première édition des Mémoires (Izbrannye proizvedeniya $v$ chesti tomakh,

T. 1. Moscou: Iskousstvo, 1964) n'étaient utilisés que deux petits chapitres de $M e^{\prime}-$ 
thode, alors que dans la deuxième édition (d'abord en allemand en 1984, ensuite en russe et à nouveau en allemand en 1997-1998), le volume des emprunts à Méthode s'est considérablement élargi (dans le premier volume p. 11-25, 26-44, 44-47, 132153, dans le deuxième, p. 323-331). Naoum Kleiman a déclaré à ce sujet : “Dans notre cas j'admets qu'un chercheur arrive et dise que les mémoires ne sont pas composés correctement, que certains textes sont intitulés de manière tout à fait incorrecte et qu'il faut tout éditer autrement. Bon! les manuscrits ont été conservés, nous n'avons rien changé ni à l'ordre du catalogage, ni à quoi que ce soit. Chacun peut faire la composition qu'il veut." Oleg Aronson/Naoum Kleiman, "Ouskolzaiuschiï Eisenstein "(Iskousstvo kino, n" 10, 1998, p. 73).

16. Nikolaï Marr (1864-1934), membre de l'Académie des sciences de Russie en 1912, doyen de la faculté des langues orientales de l'Université de Pétersbourg (1911 1918), puis de la faculté de sociologie (1918-1919) et directeur de l'institut de l'archéologie, fondateur et président de l'Académie d'État de l'histoire de culture matérielle de 1919 à sa mort, fondateur et président de l'institut japhétique (1921-1931) et de l'Institut du langage et de la pensée qui lui succède (1931-1934), dirigeant de la section de la linguistique matérialiste à l'Académie communiste dont Eisenstein suivit les cours à son retour du Mexique en 1932. Son activité scientifique, violemment mise en cause par les linguistes, qui la considéraient comme scientifiquement non fondée, a fait l'objet d'une violente critique par Staline en 1950 (voir Joseph Staline, Le Marxisme et les problèmes de linguistique [Moscou: Éditions en langues étrangères, 1950], rééd. Pékin : Éditions en langues étrangères, 1975) : “N. Marr a introduit dans la linguistique la thèse erronnée, non marxiste, de la langue considérée comme une superstructure" ainsi que "du caractère de classe de la langue» (Staline publia trois articles dans la Pravda des 20 juin, 4 juillet et 2 août 1950 sur la question, réunis ensuite en brochure). On repère des traits communs aux tentatives de Marr et d'Eisenstein de trouver une explication universelle à des phénomènes (linguistiques) divers.

17. On ignore quel texte de l'historien d'art Nikolaï Taraboukine (1899-1956) est visé ici par Eisenstein. Dans le livre de Werner (op. cit., p. 144 et sq., III «Teil Urtümliche Fassungweisen von Raum und Zeit "), il y a un long passage consacré aux dessins d'enfants comme phénomène de "montage" qui souligne, à l'aide de changements des proportions réelles "le sens important". Eisenstein donne cet exemple dans Disney (p. 261 de l'édition russe).

18. Décor d'Eisenstein de 1922, pendant son travail aux Cours supérieurs de mise en scène de Vsevolod Meyerhold, pour la pièce de George Bernard Shaw.

19. Dans cet ouvrage, Lucien confronte la vie de Rome et de la Grèce en tant que formations socio-culturelles. L'intérêt d'Eisenstein pour la vie des fourmis et leurs formes d'organisation (par la suite, il cite beaucoup le livre de Girod sur ce sujet) coïncide avec la mode pour de telles allégories des problèmes sociologiques dans les discours scientifiques (Herbert Spencer). Eisenstein évoque non seulement les fourmis, mais les vers, les amibes et les araignées pour expliquer certains moyens de perception, de motricité et les formes d'organisation sociale, en les confrontant aux procédés formels.

20. En 1928, Eisenstein projetait d'écrire une étude sur le pathos, à partir des Rougon-Macquart de Zola, après ses séminaires avec les étudiants du Collège technique d'État pour le cinéma, à qui il donnait à étudier Zola et ses procédés d'élaboration du matériau (par exemple les scènes d'orgasme et de mort, situées chaque fois dans un milieu particulier et données comme pars pro toto). Eisenstein évoque une particularité de la vision des araignées qui ne perçoivent leur proie qu'en tant que partie du fond. Werner développe ce sujet dans son livre (op. cit., chapitre "Über urtümliche seelische Verhaitungsweisen. Der diffuse Charakter urtümlicher Handlungsabläufe») en se référant aux travaux de Volkelt, Baltzer, Hempelmann, Katz. L'araignéc 
était en ce sens considérée comme exemple de la Ganzheitlichkeit, l'intégralité de l'action. L'étude des principes de composition chez Zola devait se fonder sur la confrontation de ces données. Des extraits ont été publiés dans Voprosy literatoury, $n^{\circ} 1$ (1968) et d'une manière plus détaillée dans le livre de M. Vladimirova (La Littérature mondiale et les leçons de réalisateur de S. M. Eisenstein, Moscou: Institut d'État de Moscou pour la culture, 1990 , p. 72-82).

21. Conception du "mouvement en retrait» dans la biomécanique de Meyerhold qui consiste en un petit mouvement dans la direction inverse au mouvement principal. Eisenstein disait que cette conception était le principe de base de sa "bimécanique "et l'envisageait comme analogique à la loi dialectique de la négation de la négation, que l'on peut représenter graphiquement ainsi : non pas A-B mais C-A-B.

22. Cf. la conférence de 1935, "Nouveaux problèmes de la forme filmique " (réélaborée par la suite pour constituer un chapitre de Méthode) : "Quand une femme polynésienne doit accoucher, la règle veut que toutes les entrées de la bourgade soient ouvertes, toutes les portes soient grandes ouvertes, que toute la population (y compris les hommes) ôte ceintures, tabliers, bandeaux, que tous les nœuds soient défaits, etc., c'est-à-dire que le milieu, en tous ses éléments concomitants, doit être conformé, sous un seul et même signe, au thème principal de ce qui a lieu : tout doit être ouvert, dénoué, pour faciliter au maximum la venue au monde du bébé." (1976a, p. 154). Cet exemple provient du livre de Heinz Werner (op. cit.).

23. Tiré du livre de J. Frazer où il exemplifie le transfert d'un détail d'une situation à une autre avec une conclusion erronée du point de vue logique. Voir Régissoura (Izbrannye proizvedeniẗa, vol. IV, p. 444): "Quand les maris vont à la chasse aux sangliers, personne dans la bourgade n'a le droit de mouiller ses mains ou de toucher de l'huile. Sinon les do gts des chasseurs peuvent se relâcher ou leurs paumes devenir glissantes, et le sanglier leur glisser entre les mains. Tout le monde participe à la chasse même s'il ne fait pas partie de l'expédition proprement dite."

24. "Prenons, par exemple, le procédé artistique le plus commun, qu'on appelle la pars pro toto. Son efficacité est connue de tous. Le pince-nez du médecin du Cuirassé Potemkine a marqué la mémoire de tous les spectateurs. Ce procédé consiste à remplacer le tout (le médecin) par la partie (le pince-nez) qui joue son rôle, et qui se trouve le jouer avec plus d'intensité, de sensibilité que n'en aurait eu l'exposition du tout (du médecin). Il se trouve que ce procédé est l'un des exemples les plus caractéristiques des formes mentales appartenant à la pensée primitive. À ce stade, il n'y a pas encore de représentation de l'unité du particulier et du tout au sens où nous l'entendons maintenant" (1976a, p. 151).

25. S. V. Chéréchevski, médium et hypnotiseur, possédait une mémoire prodigieuse et avait la faculté de percevoir le son comme une couleur. Il fut l'objet de nombreuses expériences de la part du psychologue Alexandre Louria, auxquelles Eisenstein a parfois assisté. Ces facultés paranormales sont décrites dans un livre de Viatcheslav Ivanov (Pair et impair. L'asymétrie du cerveau et les systèmes de signes, Moscou: Naouka, 1980).

26. Henry Irving (1838-1905), comédien anglais. Eisenstein cite fréquemment le livre que Gordon Craig lui a consacré (Henry Irving, New York, Toronto, 1930) dans un des chapitres de Méthode consacré au jeu du comédien. D'autres exemples concernent Yvette Guilbert, Davydov, Stanislavski et Meyerhold. Dans une des citations de G. Craig on lit: "Avant toute phrase ou mot, Irving faisait quelque chose de telle sorte qu'il ne puisse pas y avoir de doute pour le spectateur quant au sens auquel tendait cette phrase ou ce mot» (p. 191).

27. Terme de Meyerhold qui désigne la transmission du contenu d'une réplique par la pantomime avant la parole. Elle fut appliquée à la mise en scène de Maître Boubous d'Alexandre Faïko. 
28. René-Charles Guilbert de Pixérécourt (1773-1844), auteur de mélodrames à grand spectacle ou historiques toujours accompagnés de musique.

29. Dans le chapitre de Méthode consacré au "mouvement expressif», Eisenstein cite un livre d'Ernst Kretschmer (Medizinische Psychologie, Leipzig: 1922; dans sa bibliothèque se trouve l'édition de 1926, p. 148, 150). Werner se réfere également souvent à ce même livre: "[La femme hystérique] agit comme une poule apeurée derrière une clôture étroite: elle commence à caqueter, battre des ailes, rouler et résister jusqu'au moment où, grâce à cet excès de mouvements, elle se retrouve devant une faille de la clôture par laquelle elle peut s'enfuir."

30. Allusion au film de D. W. Griffith, Orphans of the Storm (1921), d'après Les Deux Orphelines d'Adolphe Dennery et Eugène Cormon datant de la Révolution de 1789 et dont le sujet repose sur plusieurs retrouvailles ratées des deux héroünes qui servent à retarder le dénouement et allonger l'action. Eisenstein pensait que ce mélodrame présentait l'exemple idéal de l'hystérique, décrite par Kretschmer dans sa Psychologie médicale.

31. Fameuse "ciné-phrase" du Cuirassé Potemkine.

32. Fameuse "ciné-phrase" d'Octobre introduite par le carton «Au nom de Dieu, du tzar et de la patrie" qui présente les dieux de diffétentes religions, filmés au musée d'ethnographie de Léningrad.

33. Dans sa conférence de 1935, Eisenstein utilise un exemple emprunté à Wilheim Wundt, pour expliciter cette notion: "Wundt donne dans Elemente der Völkerpsychologie des exemples de la manière dont s'expliquent des expressions figées auxquelles nous sommes habitués [...]. Ainsi la phrase: "Le Bochiman fut d'abord reçu aimablement par le Blanc pour qu'il fasse paître ses brebis; puis le Blanc a frappé le Bochiman et celui-ci s'est enfui." Cette pensée simple (et situation très fréquente compte tenu des mœurs coloniales) reçoit approximativement la forme suivante dans la langue des Bochimans: "Bochiman — là-bas - aller; ici - aller - vers - Blanc; Blanc - donner - tabac; Bochiman - aller - fumer, aller - remplir - tabac sac; Blanc - donner - viande - Bochiman; Bochiman - aller - manger viande; se lever - aller - maison; aller — gaiement, aller — s'arrêter; paître brebis — du Blanc; Blanc - venir - frapper - Bochiman; Bochiman - crier fort - mal ; Bochiman - aller — courir - loin Blanc; Blanc - courir — après Bochiman..." Ce qui frappe, c'est cette suite d'images singulières, intuitives, proches d'une suite asyntaxique. Mais si nous nous imaginons de représenter sur scène ces deux lignes de la situation initiale, nous verrons, à notre grande surprise, que nous allons construire quelque chose de très proche de cet échantillon de construction Bochiman. Et ce "quelque chose" tout aussi asyntaxique sera... un découpage de montage, qui nous est familier. C'est-à-dire retourner au "fait" que la notion avait rendu abstrait, en arrière, dans la chaîne des actions concrètes et singulières, c'est en quoi consiste le processus de transformation des paroles en actions. Les mots "il le fuit", en bochiman, seront représentés en une phrase orthodoxe de montage composée de deux morceaux : "Bochiman court", "Blanc court" - c'est un embryon de montage de "courses poursuites" à l'américaine!" (1976a, p. 156 [trad. modifiée]).

34. Il s'agit de la célèbre description de l'opéra du point de vue de Natacha Rostova dans Guerre et Paix, de Tolstoï, quand l'héroïne refuse d'accepter la convention du théâtre et voit sur scène, au lieu d'une forêt, un morceau de bois peint. V. Chklovski cite cet exemple dans "L'Art comme procédé" (1913) à côté de "Kholstomer " auquel il se réfere également pour fonder son hypothèse de l'estrangement (voir $\mathrm{T}$. Todorov (direction), Théorie de la littérature, Paris: Seuil, 1965, p. 87-90 et p. 85). Eisenstein trouvait cette explication de l'impact de l'art très peu convaincante et proposait dans le chapitre "Natacha" de Méthode son point de vue sur le mécanisme décrit de la "naturalisation" de la convention en art. 
35. "Kholstomer" (1863-1886), nouvelle de Léon Tolstoï où la perspective narrative est attribuée à un cheval.

36. Personnages du folklore russe.

37. Allusion probable au livre de Karl Storck, édité en russe sous le titre Système de Dalcroze par R. Varchavskaïa et N. Lévinskaïa, enseignantes de l'Institut du rythme de Léningrad en 1924.

38. Voir la conférence de 1935 où Eisenstein cite une réflexion d'I. Mandelstam ( $D u$ caractère du style de Gogol, 1902, p. 118) à propos d'une phrase du Tarass Boulba de Gogol: "Boulba haussa les épaules et séloigna vers son détachement. Les dernières lignes, dans la version définitive, sonnent ainsi : Il haussa les épaules Tarass Boulba, sétonna de la nature juive vive et séloigna vers le campement. Mandelstam remarque que l'effet est plus frappant en raison de la position du verbe devant le sujet. [...] il se réfere à Herbert Spencer (Essay:) qui explique la plus grande efficacité de cette interversion par l'"économie de l'attention". Il n'explique cependant pas pourquoi il est plus "économique" de procéder ainsi. Il le fait moins encore quand il s'efforce d'expliquer la même chose dans le cas cité de Tarass: "Par ce changement un contenu nouveau est introduit, absent de la version précédente: c'est l'expression de quelque chose de subjectif de la part de Tarass... Haussa les épaules Tarass Boulba, sétonna de la nature juive vive et séloigna vers ie campement - et tout est plus expressif." Nous ne pouvons tomber d'accord avec lui que sur ces derniers mots. Mais il explique l'effet au moyen de la chose même qu'il fallait expliquer! Que la deuxième version (définitive) soit sensiblement plus expressive, comme dans la plupart des cas où le verbe précède le sujet, c'est évident. Mais la raison tient, une fois encore, à ce que cette interversion correspond à la nature des formes primitives de pensée dont nous avons déjà parlé " (1976a, p. 157158 [trad. modifiée]).

39. Philosophy of Siyle [La philosophie du style] (Boston: Allyn and Bacon, 1892) de Herbert Spencer a été diffusé en Russie par Alexandre Vésélovski (EEuvres, vol. 1, $\mathrm{SPb} ., 1893$, p. 445); Chklovski le cite (d'après Vésélovski) dans "L'Art comme procédé" (op. cit.).

40. Phénomène également analysé en détail par Werner.

41. Voir Disney.

42. Eisenstein se réfere à cet exemple des Voyages de Gulliver de Jonathan Swift dans le chapitre "Pair-impair": les sages de Laputa refusant d'employer les mots communiquent en se montrant des objets qu'ils portent avec eux dans des sacs $(1980$, p. 188189).

43. Eisenstein relève dans La Pensée primitive de Lévy-Bruhl: «Les Indiens Clamates n'ont pas de terme général pour désigner la notion de renard, d'écureuil, de papillon, etc., mais toute espèce de renards, d'écureuils, etc. a son nom particulier" (édition russe, Moscou, 1930, p. 114).

44. Eisenstein veut sans doute parler de Pi Sheng, alchimiste chinois qui inventa vers 1041-1048 le premier alphabet démontable.

45. Développé dans le chapitre sur le « Roman policier» (1987, p. 57-82).

46. Armand Quatrefages de Bréau [Jean-Louis Armand de Quatrefages (18101892)], Charles Dairwin et ses précurseurs français; étude sur le transformisme (Paris: 1870). Dans l'ébauche de la préface à Méthode, Eisenstein essayait de trouver les exemples de structure sociale décrite par Karl Marx à travers une métaphore "animale». "Et réellement, il se trouve que cette conception est la reproduction de la tradition très profonde qui remonte ou plutôt descend jusqu'au stade le plus bas de la vie des animaux des formes les plus basses. Il s'agit ici de la famille des vers plats et des phénomènes desdites colonies linéaires* qui consiste en ceci. Quand, par exemple, Mieros forum lineare arrive à un certain degré de développement, son corps se serre au 
milieu. Sur la partie de l'arrière qui s'est ainsi formée sur l'extrémité la plus en arrière apparaît une deuxième bouche. Et la partie de l'arrière, sans se séparer de celle de devant, commence à se développer en une autre unité indépendante. La partie du devant se développe aussi jusqu'à l'organisme indépendant. Ainsi l'organisme de départ a produit deux nouveaux organismes indépendants mais inséparables. Chacun des nouveaux organismes parus se comporte de la même manière. D'où surgit déjà une chaîne de quatre organismes indépendants liés. Cette chaîne se dédouble de la même manière encore une fois, après quoi les huit chaînons apparus se séparent de la chaîne commune en s'arrachant l'un de l'autre et en commençant une vie indépendante. Quand il sagit de vers parasites, plats ou en rouleaux, la quantité de relles individualités liées et indépendantes arrive à un plus grand nombre. C'est le cas de Toenia. Le même phénomène est observé chez les anémides maritimes. De Quatrefages a étudié et décrit en détail Syllis prolifera qui se forme de la même manière comme l'organisme unique avec une série naissante des têtes intermédiaires. À mesure de la maturité sexuelle ces unités indépendantes s'arrachent et commencent à vivre pour leur propre compte. Chez les protozoaires (Protozoa), en particulier de type flogellata (a des cirres) il y a encore une deuxième érape. Les unités libres qui se libèrent de telle façon se rassemblent dans les colonies libres et mobiles. Là, elles s'entretissent et s'unissent encore au moyen d'une substance collante qu'elles sécrètent, et leurs cirres qui se groupent vers la périphérie servent de moyen de déplacement rotatif de toutes les colonies. Ainsi sont, par exemple, les espèces Volvox et stephanos Phoera. Ces phénomènes sont très intéressants parce qu'ils forment les étapes intermédiaires entre les structures les plus simples à travers les structures coloniales aux organismes complexes proprement dits. Dans de telles "colonies" a lieu un "partage de travail" inévitable." (f. 1923, op. 2, ed. khr. 231).

47. Paul Girod, Les Sociétés chez les animaux (Paris: Librairie J.-B. Baillère et Fils, 1891). Cf. Ia note 19 supra.

48. En 1928, Eisenstein prévoyait de répondre aux critiques d'Octobre émanant du cercle de l'école formelle (Ossip Brik, Victor Chklovski, Viktor Pertsov) qui lui reprochaient son incompréhension des lois régissant les rapports entre les métaphores verbales et visuelles (cf. Novyi Lef, 1928, n" 4, p. 29-36).

49. Ostranienie, l'étrangéification, la mise à distance, la distanciation.

50. Conan Doyle est analysé dans le chapitre «Sur le roman policier" (1987, p. 9394).

51. La triade développée et la triade enveloppée désignent le processus de la négation de la négation au sens dialectique et "la formule de la logique de la vie courante" (cf. F. Engels).

52. Le delta grec ou D russe abrège le mot "dialectique " dans les notes d'Eisenstein. 53. Il s'agit de la pièce homonyme d'Alexandre Afinogénov sur les ennemis du peuple caché.

54. Références à M. Wilson Disher, Clowns and Pantomimes (London: 1925; reprint New York: B. Blom, 1968).

55. Fonds 1923, liste 2, dossier 231, feuillets 1-7.

56. Voir le texte d'Eugène Weisman dans le fascicule Les Erreurs du Pré de Béjine, Moscou, 1937.

57. Résumé de cours sur la psychologie de l'art dans: Boris Meilah et Nikolaï Khrenov (direction), Psybologija processov hudozhestvennogo tvorchestva [Psychologie des processus de la création artistique] (Leningrad: Naouka, 1980, p. 188-203); en allemand dans: Kunst und Literatur, 30. Jahregang, n"9, September 1982, p. 924-953.

58. Cette formule paraphrase celle de Staline sur l'art du réalisme socialiste: "socialiste dans le contenu, nationale dans la forme». 
59. Eisenstein vise vraisemblablement le discours de Staline au Premier Congrès des kolkhoziens-oudarniks de l'Union soviétique du 19.02.1933 qui a été édité à l'époque (Moscou: Partizdat, 1933) puis dans les Euvres (Moscou: Glavnoe izdatel'stvo polititcheskoï literatoury, 1946-1951, v. 13).

60. Les deux derniers mots sont écrits en lettres latines dans le texte cyrillique.

61. Fonds 1923, liste 2, dossier 236.

62. Il avait alors pu disposer d'un wagon de chemin de fer pour transporter avec lui sa bibliothèque. L'essentiel de ses livres resta cependant à Moscou comme les matériaux du livre.

63. Karl Holleck-Weitmann, Nordische Religion und nordische Kunst (Berlin : Struppe \& Winckler, 1935).

64. Sigle par lequel Eisenstein désigne le retour dans le ventre maternel, Mutterleibversenkung (voir Eisenstein, 1999a).

65. Voir l'étude "Ermolova" (1980, p. 220-248).

66. Voir "L'organique et le pathétique" (1976b, p. 72-78) où ce tableau de Sourikov est analysé.

67. Eisenstein parle de la nouvelle de David Herbert Lawrence (1885-1930) St. Mawr, qui a été éditée à part à New York, chez A. A. Knopf, 1925, et qu'il comparait à « Kholstomer " de Tolstoï.

68. Il s'agit d'Agnia Kasatkina (1903), amie d'Eisenstein au début des années vingt. Il pense vraisemblablement à l'Aglaỉa de L'Idiot.

69. Boris Arvatov (1896-1943), théoricien de l'art productiviste avec lequel Eisenstein travailla au Proletkult sur la mise en scène du Mexicain. Agnia Kasatkina était sa fiancée. Souffrant de schizophrénie en raison d'une blessure à la tête, il vécut de la fin des années vingt à sa mort dans une clinique psychiatrique.

70. Ielizaveta Téléchéva (1892-1943), metteur en scène au MKhAT, assistante au VGIK et à cette époque "compagne officielle " d'Eisenstein.

71. Véra Ianoukova (1904-1939), comédienne de la troupe de Proletkult qui jouait le rôle de Mamaïeva dans la mise en scène du Sage et dont Eisenstein était amoureux.

72. Ce petit chapitre de 8 pages a été omis dans la publication des textes d'Eisenstein sur Disney.

73. Ici, omission d'une citation concernant un fragment non retenu. Voir fonds 1923, liste 2, dossier 323, feuillets 11-14.

74. Voir le compte rendu de Herbert Muschamp (New York Times Art and Leisure, 16.01.2000).

75. Oleg Aronson/Naoum Kleiman, "Ouskolzaiouschiï Eisenstein" (Iskousstvo kino, $1998, n^{\circ} 10$, p. 74). Cet entretien a reçu le prix de la meilleure publication de l'année de la revue.

76. Serguei Eisenstein, "Samoe uzhasnoe, tchem ia stradaiu, — eto bolezn' voli. Pis'ma k Pere Atachevoi " (Kinovedtcheskie zapiski, n³ 36/37, 1997/1998, p. 230).

\section{OUVRAGES CITÉS}

N.B. Les ouvrages cités d'Eisenstein se retrouvent, en fin de numéro, dans une liste particulière.

Seton, Marie. Sergei M. Eisenstein. A Biography. London: The Bodley Head, 1952.

Iampolski, Mikhaill. "Theory as Quotation", October, n” 88 (Spring 1999). 